\title{
Robust stability analysis of impulsive quaternion-valued neural networks with distributed delays and parameter uncertainties
}

\author{
Jielin Zhou' ${ }^{1}$ Yuanshun Tan ${ }^{1 *}$, Xiaofeng Chen ${ }^{1}$ and Zijian Liu ${ }^{1}$
}

"Correspondence:

tanys625@163.com

${ }^{1}$ College of Mathematics and

Statistics, Chongqing Jiaotong University, Chongqing, 400074, China

\section{说) Springer}

\begin{abstract}
In this paper, an impulsive quaternion-valued neural networks (QVNNs) model with leakage, discrete, and distributed delays is considered. Based on the homeomorphic mapping method, Lyapunov stability theorem, and linear matrix inequality (LMI) approach, sufficient conditions for the existence, uniqueness, and global robust stability of the equilibrium point of the impulsive QVNNs are provided. A numerical example is provided to confirm the obtained results. A conclusion is presented in the end.
\end{abstract}

Keywords: Quaternion-valued neural networks; Distributed delays; Global robust stability; Lyapunov stability theorem

Neural networks have been proposed in the 1940s by psychologist MuCulloch and mathematician Pitts and since then have been paid extensive attention of researchers due to their wide applications in scientific and technological fields, such as image processing, signal processing, fault diagnosis, and associative memory [1-4]. Real-valued neural network (RVNN) is the most typical representative of a neural network system, and different kinds of stability are studied by researchers, for example, the power-rate global stability of the equilibrium is proposed in [5]. In [3], the authors propose a new concept of global $\mu$-stability, which unifies the exponential stability, power-rate stability, and log-stability of neural networks. In 1992, Hirose proposed complex-valued neural networks (CVNNs) in which the state, output, weight, and domain of attraction are all complex values [6]. Compared with RVNNs, the advantage of CVNNs is that they can directly deal with 2D data. Since then, numerical researches pass the theoretical analysis of the basic properties of real- and complex-valued neurons, summarize the differences between the two, and clarify some inherent properties of complex-valued neurons at the boundary [7-14]. However, there are still some problems that the RVNNs and the CVNNs cannot deal with easily, such as 4D signals, body images, which are four- or more dimensional, new meth-

(c) The Author(s) 2020. This article is licensed under a Creative Commons Attribution 4.0 International License, which permits use, sharing, adaptation, distribution and reproduction in any medium or format, as long as you give appropriate credit to the original author(s) and the source, provide a link to the Creative Commons licence, and indicate if changes were made. The images or other third party material in this article are included in the article's Creative Commons licence, unless indicated otherwise in a credit line to the material. If material is not included in the article's Creative Commons licence and your intended use is not permitted by statutory regulation or exceeds the permitted use, you will need to obtain permission directly from the copyright holder. To view a copy of this licence, visit http://creativecommons.org/licenses/by/4.0/. 
ods or theories have to be put forward, the theory of QVNNs thus emerges as required [4].

In practical life, QVNNs have many applications in various areas. One practical application by QVNNs is the 3D geometrical affine transformation, especially spatial rotation, which can be represented efficiently and compactly by QVNNs. Other practical applications of QVNNs are image impression, color night vision, etc. [15-22]. One specific example in reality is reconstructing gray and color images using designed QVNNs, which can possess high storage capacity in applications of associative memory and pattern recognition. For this class of QVNNs, via eigenstructure method, the results developed by researchers enable us to synthesize neural networks with specified equilibrium points. Other applications of parameter uncertainty for the integer-order neural network can be seen in the design of PI controller, the stability region of systems with parameter uncertainty is essential to the design of PI controller. In fact, in the actual operation process, the measured values of the characteristics or parameters will deviate from their actual values due to the inaccurate measurement or calculation. And system parameters will be changed. The system may not be stable after the parameter changes. Once the stability is lost, the images will be difficult to reconstruct.

To a general QVNN in real life, the delay is inevitable owing to the delay of transmission line, equivalent circuit of some components, integration and communication, etc. Usually, there exit three types of common delay: leakage delay, discrete delay, and distributed delay. For a simple circuit with a small number of neurons, it can be described by a time-delay feedback system with a fixed time delay [23-26]. However, since the neural networks are composed of a lot of neurons, with a large number of parallel channels, and the neural networks have the characteristics of time and space, we can introduce the distribution delay to describe some characteristics of the neural networks. In [24], the leakage delay is introduced into the model to explore its stability. In [25, 27], the influence of leakage and discrete delays on CVNNs are studied. In [28], the effects of leakage and discrete delays in QVNNs are considered. To the best of our knowledge, the results of QVNNs with leakage, discrete, and distributed delays are few. Besides time delays, impulse also exists in the application of neural networks, which makes the model more realistic [29-32]. For example, during the implementation of an electronic network, the state of the network will be interfered by transients, and sudden changes will occur at a certain time, which may be caused by switching phenomena, frequency changes, or other accidents. Therefore, it is necessary to consider the effect of impulsive effects and delays on the dynamic behavior of neural networks.

For the neural network model with different number fields, researchers all over the world have extended the model in different directions: considering different types of time delays, such as leakage delay, discrete delay, distributed delay, etc.; considering different types of equations, such as impulsive differential equation, stochastic differential equation, etc.; to study the existence, boundedness of equilibrium of a system, different types of stability, synchronization, and bifurcation of a neural network. The research scope has also been extended from integer-order neural networks to fractional-order neural networks $[33,34]$. Recently, in [35], the authors presented a delayed QVNN with parameter uncertainties and investigated the robust stability of the system. In [36], the authors established sufficient conditions on the existence, uniqueness, and global stability of the equilibrium of a delayed QVNN with interval parameter uncertainties through constructing a couple 
of LMIs. Sequently, a sufficient criterion was obtained to ensure global robust stability of QVNNs by applying Lyapunov function method and inequality techniques. But in its judging criteria, two negative definite matrices are needed, and the terms in these matrices are the maximum values, which are determined by the absolute values of the upper and lower bounds of the elements of the connection weight matrix, ignoring the sign of the connection weight value. To overcome this shortcoming, Wang in [28] revisited the system and obtained a criterion whose elements rely on both the lower and upper bounds of the interval parameters.

Based on the above discussion, we propose a network model with mixed delays and impulsive effects. By means of quaternion-valued inequality, Lyapunov function, and homeomorphic mapping, a new sufficient condition to ensure the existence, uniqueness, and global robust stability of an equilibrium point for the QVNN is derived, which can be checked numerically using the effective YALMIP toolbox in MATLAB. Comparing to other references, our contributions lie in two aspects: firstly, the traditional model is extended in the article, the distributed delay and impulsive effect are considered in our model, which make the model more practical and is the substantial extension of the study of $[8,28]$, because the existence of the distributed delay and impulsive effect increases the dimension of the system, which produces great difficulties in numerical simulation and for the proof of the negative definiteness for $V$ function. Secondly, in the judging criteria of reference $[13,36]$, the negative definiteness of two matrices is needed, however, in this paper, only one negative definite judging matrix is enough to guarantee the existence, uniqueness, and global robust stability of the equilibrium point. Moreover, the elements of the given criterion matrix in our proposed results depend not only on the lower bounds but also on the upper bounds of the interval parameters, which is less conservative and extend some previous contributions; for details, see [13, 14, 36].

The structure of this paper is as follows. In Sect. 2, the model of impulsive neural networks with three kinds of time delays involving leakage delay, discrete delay, and distributed delay is proposed, and some basic preparations are introduced. Sufficient conditions for the existence, uniqueness, and global robust stability of equilibrium point are established in Sect. 3. To illustrate the validity of the main results, numerical simulation is carried out in Sect. 4. Finally, we make a brief conclusion at the end.

\section{Problem formulation and preliminaries}

At the beginning, we give some notations used throughout the paper before proposing our model.

Let $\mathbb{R}, \mathbb{C}$, and $\mathbb{H}$ be the real field, complex field, and the skew field of quaternions, respectively. Let $\mathbb{R}^{n}, \mathbb{C}^{n}$, and $\mathbb{H}^{n}$ be the $n$-dimensional vectors with entries from $\mathbb{R}, \mathbb{C}$, and $\mathbb{H}$, respectively; $\mathbb{R}^{n \times m}, \mathbb{C}^{n \times m}$ and $\mathbb{H}^{n \times m}$ represent $n \times m$ real-valued matrices, complexvalued matrices, and quaternion-valued matrices, respectively; $\bar{A}, A^{T}$, and $A^{*}$ denote the conjugate, transpose, and conjugate transpose of a matrix $A$, respectively. For any $z \in \mathbb{C}^{n}$, let $\|z\|=\sqrt{z^{*} z}$ be the norm of $z$. The notation $X \geq Y$ (or $X>Y$ ) means that $X-Y$ is positive semidefinite (or positive definite). For a positive definite Hermitian matrix $H, \lambda_{\max }(H)$ and $\lambda_{\min }(H)$ represent the maximum and minimum eigenvalues of $H$. Symbol $I$ denotes an identity matrix with an appropriate dimension, and symbol $*$ shows the conjugate transpose of a suitable block in a Hermitian matrix.

Next, we introduce some preliminaries on quaternion-valued notations and operations. If $p \in \mathbb{H}$, then it can be expressed as $p=p_{0}+p_{1} l+p_{2} J+p_{3} \kappa$, where $p_{0}$ is the real part of the 
quaternion, and $p_{1}, p_{2}$, and $p_{3}$ are the imaginary parts of the quaternion. When $a, b \in \mathbb{H}$ with $a=a_{0}+a_{1} l+a_{2} J+a_{3} \kappa, b=b_{0}+b_{1} l+b_{2} J+b_{3} \kappa$, then $a \preceq b$ means $a_{i} \leq b_{i}(i=1,2,3)$. Also, if $A, B \in \mathbb{H}^{n \times n}$, then $A \preceq B$ means $a_{i j} \leq b_{i j}, i, j=1,2, \ldots, n$, where $A=\left(a_{i j}\right)_{n \times n}$ and $B=\left(b_{i j}\right)_{n \times n}$.

\subsection{Model description}

In this paper, we consider the following impulsive QVNNs model with distributed delay:

$$
\left\{\begin{aligned}
\dot{q}(t)= & -D q(t-\delta)+A g(q(t))+B g(q(t-\tau)) \\
& +C \int_{t}^{+\infty} K(t-s) g(q(s)) d s+J, \quad t>0, t \neq t_{k}, \\
\Delta q\left(t_{k}\right)= & q\left(t_{k}\right)-q\left(t_{k}^{-}\right)=M_{k}\left(q\left(t_{k}^{-}\right), q_{t_{k}^{-}}\right), \quad k=1,2, \ldots
\end{aligned}\right.
$$

where $q(t)=\left(q_{1}(t), q_{2}(t), \ldots, q_{n}(t)\right)^{T} \in \mathbb{H}^{n}$ is the state vector of the neural neuron of the neural network at time $t ; g(q(t))=\left(g\left(q_{1}(t)\right), g\left(q_{2}(t)\right), \ldots, g\left(q_{n}(t)\right)\right)^{T} \in \mathbb{H}^{n}$ represents the activation function of neurons; $D=\operatorname{diag}\left(d_{1}, d_{2}, \ldots, d_{n}\right) \in \mathbb{R}^{n \times n}$ is the self-feedback connection weight matrix with $d_{j}>0(j=1,2, \ldots, n) ; A \in \mathbb{H}^{n \times n}$ is the connection weight matrix; $B \in \mathbb{H}^{n \times n}$ is the delayed connection weight matrix; $C \in \mathbb{H}^{n \times n}$ is the distributively delayed connection weight matrix; $J=\left(J_{1}, J_{2}, \ldots, J_{n}\right)^{T} \in \mathbb{H}^{n}$ denotes the input vector; $\delta>0$ refers to the leakage delay; $0<\tau<\rho$ refers to the transmission delay, and $0<t_{1}<t_{2}<\cdots$ is a strictly increasing sequence such that $\lim _{k \rightarrow \infty} t_{k}=+\infty$. Also $K(\cdot):[0,+\infty) \rightarrow[0,+\infty)$ is the delay kernel and $M_{k}$ is the impulsive function.

Assume that system (1) satisfies the initial conditions given by

$$
q(s)=\vartheta(s), \quad s \in[-\rho, 0]
$$

here $\vartheta(\cdot)$ is a quaternion field continuous function defined on $[-\rho, 0], \vartheta(s)=\left(\vartheta_{1}(s), \vartheta_{2}(s)\right.$, $\left.\ldots, \vartheta_{n}(s)\right)^{T} \in C\left([-\rho, 0], \mathbb{H}^{n}\right)$ and the norm

$$
\|\vartheta(s)\|=\sup _{s \in[-\rho, 0]} \sqrt{\sum_{i=1}^{n}\left|\vartheta_{i}(t)\right|^{2} .}
$$

This paper will require the following assumptions:

$\left(\mathbf{A}_{1}\right)$ For $i=1,2, \ldots, n$, the neuron activation function $g_{i}$ is continuous and satisfies

$$
\left|g_{i}\left(q_{1}\right)-g_{i}\left(q_{2}\right)\right| \leq l_{i}\left|q_{1}-q_{2}\right|, \quad \forall q_{1}, q_{2} \in \mathbb{H},
$$

where $l_{i}$ is a real-valued positive constant. In addition, we define $L=\operatorname{diag}\left\{L_{1}, L_{2}, \ldots, L_{n}\right\}$ for the convenience of the next proof.

$\left(\mathbf{A}_{2}\right)$ The matrices $D, C, B, A$, and $J$ in (1) belong to the following sets, respectively:

$$
\begin{aligned}
& D_{I}=\left\{D \in \mathbb{R}^{n \times n}: 0 \prec \check{D} \preceq D \preceq \hat{D}, \check{D}, \hat{D} \in \mathbb{R}^{n \times n}\right\}, \\
& C_{I}=\left\{C \in \mathbb{H}^{n \times n}: \check{C} \preceq C \preceq \hat{C}, \check{C}, \hat{C} \in \mathbb{H}^{n \times n}\right\}, \\
& B_{I}=\left\{B \in \mathbb{H}^{n \times n}: \check{B} \preceq B \preceq \hat{B}, \check{B}, \hat{B} \in \mathbb{H}^{n \times n}\right\}, \\
& A_{I}=\left\{A \in \mathbb{H}^{n \times n}: \check{A} \preceq A \preceq \hat{A}, \check{A}, \hat{A} \in \mathbb{H}^{n \times n}\right\}, \\
& J=\left\{J \in \mathbb{H}^{n}: \check{J} \preceq J \preceq \hat{J}, \check{J}, \hat{J} \in \mathbb{H}^{n}\right\},
\end{aligned}
$$


where $\check{D}=\operatorname{diag}\left\{\check{d}_{1}, \check{d}_{2}, \ldots, \check{d}_{n}\right\}, \hat{D}=\operatorname{diag}\left\{\hat{d}_{1}, \hat{d}_{2}, \ldots, \hat{d}_{n}\right\}, \check{C}=\left(\check{c}_{i j}\right)_{n \times n}, \hat{C}=\left(\hat{c}_{i j}\right)_{n \times n}, \check{B}=\left(\check{b}_{i j}\right)_{n \times n}$, $\hat{B}=\left(\hat{b}_{i j}\right)_{n \times n}, \check{A}=\left(\check{a}_{i j}\right)_{n \times n}, \hat{A}=\left(\hat{a}_{i j}\right)_{n \times n}$.

$\left(\mathbf{A}_{3}\right) K$ is a real-valued nonnegative continuous function defined on $[0,+\infty]$ and satisfies

$$
\int_{0}^{+\infty} K(s) d s=1, \quad \int_{0}^{+\infty} s K(s) d s<+\infty
$$

For every $A_{i}=A_{i}^{R}+\imath A_{i}^{I}+\jmath A_{i}^{J}+\kappa A_{i}^{K}, B_{i}=B_{i}^{R}+\imath B_{i}^{I}+\jmath B_{i}^{J}+\kappa B_{i}^{K}, C_{i}=C_{i}^{R}+\imath C_{i}^{I}+\jmath C_{i}^{J}+\kappa C_{i}^{K} \in$ $\mathbb{H}^{n \times n}, i=0,1$ let

$$
\begin{aligned}
& A_{0}^{R}=\frac{1}{2}\left(\hat{A}^{R}+\check{A}^{R}\right), \quad A_{1}^{R}=\frac{1}{2}\left(\hat{A}^{R}-\check{A}^{R}\right)=\left(\phi_{i j}^{R}\right)_{n \times n^{\prime}} \\
& A_{0}^{I}=\frac{1}{2}\left(\hat{A}^{I}+\check{A}^{I}\right), \quad A_{1}^{I}=\frac{1}{2}\left(\hat{A}^{I}-\check{A}^{I}\right)=\left(\phi_{i j}^{I}\right)_{n \times n}, \\
& A_{0}^{J}=\frac{1}{2}\left(\hat{A}^{J}+\check{A}^{J}\right), \quad A_{1}^{J}=\frac{1}{2}\left(\hat{A}^{J}-\check{A}^{J}\right)=\left(\phi_{i j}^{J}\right)_{n \times n^{\prime}} \\
& A_{0}^{K}=\frac{1}{2}\left(\hat{A}^{K}+\check{A}^{K}\right), \quad A_{1}^{K}=\frac{1}{2}\left(\hat{A}^{K}-\check{A}^{K}\right)=\left(\phi_{i j}^{K}\right)_{n \times n^{\prime}} \\
& B_{0}^{R}=\frac{1}{2}\left(\hat{B}^{R}+\check{B}^{R}\right), \quad B_{1}^{R}=\frac{1}{2}\left(\hat{B}^{R}-\check{B}^{R}\right)=\left(\varphi_{i j}^{R}\right)_{n \times n^{\prime}} \\
& B_{0}^{I}=\frac{1}{2}\left(\hat{B}^{I}+\check{B}^{I}\right), \quad B_{1}^{I}=\frac{1}{2}\left(\hat{B}^{I}-\check{B}^{I}\right)=\left(\varphi_{i j}^{I}\right)_{n \times n^{\prime}} \\
& B_{0}^{J}=\frac{1}{2}\left(\hat{B}^{J}+\check{B}^{J}\right), \quad B_{1}^{J}=\frac{1}{2}\left(\hat{B}^{J}-\check{B}^{J}\right)=\left(\varphi_{i j}^{J}\right)_{n \times n^{\prime}} \\
& B_{0}^{K}=\frac{1}{2}\left(\hat{B}^{K}+\check{B}^{K}\right), \quad B_{1}^{K}=\frac{1}{2}\left(\hat{B}^{K}-\check{B}^{K}\right)=\left(\varphi_{i j}^{K}\right)_{n \times n^{\prime}} \\
& C_{0}^{R}=\frac{1}{2}\left(\hat{C}^{R}+\check{C}^{R}\right), \quad C_{1}^{R}=\frac{1}{2}\left(\hat{C}^{R}-\check{C}^{R}\right)=\left(\psi_{i j}^{R}\right)_{n \times n}, \\
& C_{0}^{I}=\frac{1}{2}\left(\hat{C}^{I}+\check{C}^{I}\right), \quad C_{1}^{I}=\frac{1}{2}\left(\hat{C}^{I}-\check{C}^{I}\right)=\left(\psi_{i j}^{I}\right)_{n \times n}, \\
& C_{0}^{J}=\frac{1}{2}\left(\hat{C}^{J}+\check{C}^{J}\right), \quad C_{1}^{J}=\frac{1}{2}\left(\hat{C}^{J}-\check{C}^{J}\right)=\left(\psi_{i j}^{J}\right)_{n \times n}, \\
& C_{0}^{K}=\frac{1}{2}\left(\hat{C}^{K}+\check{C}^{K}\right), \quad C_{1}^{K}=\frac{1}{2}\left(\hat{C}^{K}-\check{C}^{K}\right)=\left(\psi_{i j}^{K}\right)_{n \times n}, \\
& D_{0}=\frac{1}{2}(\hat{D}+\check{D}) \text {, } \\
& D_{1}=\frac{1}{2}(\hat{D}-\check{D})=\left(\eta_{i j}\right)_{n \times n}=\frac{1}{2} \operatorname{diag}\left\{\hat{d}_{1}-\check{d}_{1}, \hat{d}_{1}-\check{d}_{2}, \ldots, \hat{d}_{n}-\check{d}_{n}\right\} .
\end{aligned}
$$

In addition, $A_{1}^{X} \succeq 0, B_{1}^{X} \succeq 0, C_{1}^{X} \succeq 0$, and $D_{1} \succeq 0$ with $X$ expressing $R, I, J, K$, respectively. Let $e_{i}=(0,0, \ldots, 1, \ldots, 0)_{n \times 1}^{T}$ be the vector with the $i$ th entry 1 . We define:

$$
\begin{aligned}
U_{A}^{R} & =\left(\sqrt{\phi_{11}^{R}} e_{1}, \ldots, \sqrt{\phi_{1 n}^{R}} e_{1}, \sqrt{\phi_{21}^{R}} e_{2}, \ldots, \sqrt{\phi_{2 n}^{R}} e_{2}, \ldots, \sqrt{\phi_{n 1}^{R}} e_{n}, \ldots, \sqrt{\phi_{n n}^{R}} e_{n}\right)_{n \times n^{2}}, \\
U_{A}^{I} & =\left(\sqrt{\phi_{11}^{I}} e_{1}, \ldots, \sqrt{\phi_{1 n}^{I}} e_{1}, \sqrt{\phi_{21}^{I}} e_{2}, \ldots, \sqrt{\phi_{2 n}^{I}} e_{2}, \ldots, \sqrt{\phi_{n 1}^{I}} e_{n}, \ldots, \sqrt{\phi_{n n}^{I}} e_{n}\right)_{n \times n^{2}}, \\
U_{A}^{J} & =\left(\sqrt{\phi_{11}^{I}} e_{1}, \ldots, \sqrt{\phi_{1 n}^{I}} e_{1}, \sqrt{\phi_{21}^{I}} e_{2}, \ldots, \sqrt{\phi_{2 n}^{J}} e_{2}, \ldots, \sqrt{\phi_{n 1}^{I}} e_{n}, \ldots, \sqrt{\phi_{n n}^{I}} e_{n}\right)_{n \times n^{2}}, \\
U_{A}^{K} & =\left(\sqrt{\phi_{11}^{K}} e_{1}, \ldots, \sqrt{\phi_{1 n}^{K}} e_{1}, \sqrt{\phi_{21}^{K}} e_{2}, \ldots \sqrt{\phi_{2 n}^{K}} e_{2}, \ldots, \sqrt{\phi_{n 1}^{K}} e_{n}, \ldots, \sqrt{\phi_{n n}^{K}} e_{n}\right)_{n \times n^{2}},
\end{aligned}
$$


Chou et al. Advances in Difference Equations

(2021) 2021:12

Page 6 of 33

$$
\begin{aligned}
& U_{B}^{R}=\left(\sqrt{\varphi_{11}^{R}} e_{1}, \ldots, \sqrt{\varphi_{1 n}^{R}} e_{1}, \sqrt{\varphi_{21}^{R}} e_{2}, \ldots, \sqrt{\varphi_{2 n}^{R}} e_{2}, \ldots, \sqrt{\varphi_{n 1}^{R}} e_{n}, \ldots, \sqrt{\varphi_{n n}^{R}} e_{n}\right)_{n \times n^{2}}, \\
& U_{B}^{I}=\left(\sqrt{\varphi_{11}^{I}} e_{1}, \ldots, \sqrt{\varphi_{1 n}^{I}} e_{1}, \sqrt{\varphi_{21}^{I}} e_{2}, \ldots, \sqrt{\varphi_{2 n}^{I}} e_{2}, \ldots, \sqrt{\varphi_{n 1}^{I}} e_{n}, \ldots, \sqrt{\varphi_{n n}^{I}} e_{n}\right)_{n \times n^{2}}, \\
& U_{B}^{J}=\left(\sqrt{\varphi_{11}^{J}} e_{1}, \ldots, \sqrt{\varphi_{1 n}^{J}} e_{1}, \sqrt{\varphi_{21}^{J}} e_{2}, \ldots, \sqrt{\varphi_{2 n}^{J}} e_{2}, \ldots, \sqrt{\varphi_{n 1}^{J}} e_{n}, \ldots, \sqrt{\varphi_{n n}^{J}} e_{n}\right)_{n \times n^{2}}, \\
& U_{B}^{K}=\left(\sqrt{\varphi_{11}^{K}} e_{1}, \ldots, \sqrt{\varphi_{1 n}^{K}} e_{1}, \sqrt{\varphi_{21}^{K}} e_{2}, \ldots, \sqrt{\varphi_{2 n}^{K}} e_{2}, \ldots, \sqrt{\varphi_{n 1}^{K}} e_{n}, \ldots, \sqrt{\varphi_{n n}^{K}} e_{n}\right)_{n \times n^{2}}, \\
& U_{C}^{R}=\left(\sqrt{\psi_{11}^{R}} e_{1}, \ldots, \sqrt{\psi_{1 n}^{R}} e_{1}, \sqrt{\psi_{21}^{R}} e_{2}, \ldots, \sqrt{\psi_{2 n}^{R}} e_{2}, \ldots, \sqrt{\psi_{n 1}^{R}} e_{n}, \ldots, \sqrt{\psi_{n n}^{R}} e_{n}\right)_{n \times n^{2}}, \\
& U_{C}^{I}=\left(\sqrt{\psi_{11}^{I}} e_{1}, \ldots, \sqrt{\psi_{1 n}^{I}} e_{1}, \sqrt{\psi_{21}^{I}} e_{2}, \ldots, \sqrt{\psi_{2 n}^{I}} e_{2}, \ldots, \sqrt{\psi_{n 1}^{I}} e_{n}, \ldots, \sqrt{\psi_{n n}^{I}} e_{n}\right)_{n \times n^{2}} \text {, } \\
& U_{C}^{J}=\left(\sqrt{\psi_{11}^{J}} e_{1}, \ldots, \sqrt{\psi_{1 n}^{J}} e_{1}, \sqrt{\psi_{21}^{J}} e_{2}, \ldots, \sqrt{\psi_{2 n}^{J}} e_{2}, \ldots, \sqrt{\psi_{n 1}^{J}} e_{n}, \ldots, \sqrt{\psi_{n n}^{J}} e_{n}\right)_{n \times n^{2}}, \\
& U_{C}^{K}=\left(\sqrt{\psi_{11}^{K}} e_{1}, \ldots, \sqrt{\psi_{1 n}^{K}} e_{1}, \sqrt{\psi_{21}^{K}} e_{2}, \ldots, \sqrt{\psi_{2 n}^{K}} e_{2}, \ldots, \sqrt{\psi_{n 1}^{K}} e_{n}, \ldots, \sqrt{\psi_{n n}^{K}} e_{n}\right)_{n \times n^{2}} \text {, } \\
& U_{D}=\left(\sqrt{\eta_{11}} e_{1}, \ldots, \sqrt{\eta_{1 n}} e_{1}, \sqrt{\eta_{21}} e_{2}, \ldots, \sqrt{\eta_{2 n}} e_{2}, \ldots, \sqrt{\eta_{n 1}} e_{n}, \ldots, \sqrt{\eta_{n n}} e_{n}\right)_{n \times n^{2}}, \\
& V_{A}^{R}=\left(\sqrt{\phi_{11}^{R}} e_{1}, \ldots, \sqrt{\phi_{1 n}^{R}} e_{n}, \sqrt{\phi_{21}^{R}} e_{1}, \ldots, \sqrt{\phi_{2 n}^{R}} e_{n}, \ldots, \sqrt{\phi_{n 1}^{R}} e_{1}, \ldots, \sqrt{\phi_{n n}^{R}} e_{n}\right)_{n^{2} \times n^{T}}^{T} \text {, } \\
& V_{A}^{I}=\left(\sqrt{\phi_{11}^{I}} e_{1}, \ldots, \sqrt{\phi_{1 n}^{I}} e_{n}, \sqrt{\phi_{21}^{I}} e_{1}, \ldots, \sqrt{\phi_{2 n}^{I}} e_{n}, \ldots, \sqrt{\phi_{n 1}^{I}} e_{1}, \ldots, \sqrt{\phi_{n n}^{I}} e_{n}\right)_{n^{2} \times n}^{T} \text {, } \\
& V_{A}^{J}=\left(\sqrt{\phi_{11}^{J}} e_{1}, \ldots, \sqrt{\phi_{1 n}^{J}} e_{n}, \sqrt{\phi_{21}^{J}} e_{1}, \ldots, \sqrt{\phi_{2 n}^{J}} e_{n}, \ldots, \sqrt{\phi_{n 1}^{J}} e_{1}, \ldots, \sqrt{\phi_{n n}^{J}} e_{n}\right)_{n^{2} \times n^{\prime}}^{T} \text {, } \\
& V_{A}^{K}=\left(\sqrt{\phi_{11}^{K}} e_{1}, \ldots, \sqrt{\phi_{1 n}^{K}} e_{n}, \sqrt{\phi_{21}^{K}} e_{1}, \ldots, \sqrt{\phi_{2 n}^{K}} e_{n}, \ldots, \sqrt{\phi_{n 1}^{K}} e_{1}, \ldots, \sqrt{\phi_{n n}^{K}} e_{n}\right)_{n^{2} \times n}^{T} \text {, } \\
& V_{B}^{R}=\left(\sqrt{\varphi_{11}^{R}} e_{1}, \ldots, \sqrt{\varphi_{1 n}^{R}} e_{n}, \sqrt{\varphi_{21}^{R}} e_{1}, \ldots, \sqrt{\varphi_{2 n}^{R}} e_{n}, \ldots, \sqrt{\varphi_{n 1}^{R}} e_{1}, \ldots, \sqrt{\varphi_{n n}^{R}} e_{n}\right)_{n^{2} \times n^{\prime}}^{T} \text {, } \\
& V_{B}^{I}=\left(\sqrt{\varphi_{11}^{I}} e_{1}, \ldots, \sqrt{\varphi_{1 n}^{I}} e_{n}, \sqrt{\varphi_{21}^{I}} e_{1}, \ldots, \sqrt{\varphi_{2 n}^{I}} e_{n}, \ldots, \sqrt{\varphi_{n 1}^{I}} e_{1}, \ldots, \sqrt{\varphi_{n n}^{I}} e_{n}\right)_{n^{2} \times n}^{T} \text {, } \\
& V_{B}^{J}=\left(\sqrt{\varphi_{11}^{J}} e_{1}, \ldots, \sqrt{\varphi_{1 n}^{J}} e_{n}, \sqrt{\varphi_{21}^{J}} e_{1}, \ldots, \sqrt{\varphi_{2 n}^{J}} e_{n}, \ldots, \sqrt{\varphi_{n 1}^{J}} e_{1}, \ldots, \sqrt{\varphi_{n n}^{J}} e_{n}\right)_{n^{2} \times n}^{T} \text {, } \\
& V_{B}^{K}=\left(\sqrt{\varphi_{11}^{K}} e_{1}, \ldots, \sqrt{\varphi_{1 n}^{K}} e_{n}, \sqrt{\varphi_{21}^{K}} e_{1}, \ldots, \sqrt{\varphi_{2 n}^{K}} e_{n}, \ldots, \sqrt{\varphi_{n 1}^{K}} e_{1}, \ldots, \sqrt{\varphi_{n n}^{K}} e_{n}\right)_{n^{2} \times n^{2}}^{T}, \\
& V_{C}^{R}=\left(\sqrt{\psi_{11}^{R}} e_{1}, \ldots, \sqrt{\psi_{1 n}^{R}} e_{n}, \sqrt{\psi_{21}^{R}} e_{1}, \ldots, \sqrt{\psi_{2 n}^{R}} e_{n}, \ldots, \sqrt{\psi_{n 1}^{R}} e_{1}, \ldots, \sqrt{\psi_{n n}^{R}} e_{n}\right)_{n^{2} \times n^{2}}^{T} \text {, } \\
& V_{C}^{I}=\left(\sqrt{\psi_{11}^{I}} e_{1}, \ldots, \sqrt{\psi_{1 n}^{I}} e_{n}, \sqrt{\psi_{21}^{I}} e_{1}, \ldots, \sqrt{\psi_{2 n}^{I}} e_{n}, \ldots, \sqrt{\psi_{n 1}^{I}} e_{1}, \ldots, \sqrt{\psi_{n n}^{I}} e_{n}\right)_{n^{2} \times n}^{T} \text {, } \\
& V_{C}^{J}=\left(\sqrt{\psi_{11}^{J}} e_{1}, \ldots, \sqrt{\psi_{1 n}^{J}} e_{n}, \sqrt{\psi_{21}^{J}} e_{1}, \ldots, \sqrt{\psi_{2 n}^{J}} e_{n}, \ldots, \sqrt{\psi_{n 1}^{J}} e_{1}, \ldots, \sqrt{\psi_{n n}^{J}} e_{n}\right)_{n^{2} \times n^{\prime}}^{T} \text {, } \\
& V_{C}^{K}=\left(\sqrt{\psi_{11}^{K}} e_{1}, \ldots, \sqrt{\psi_{1 n}^{K}} e_{n}, \sqrt{\psi_{21}^{K}} e_{1}, \ldots, \sqrt{\psi_{2 n}^{K}} e_{n}, \ldots, \sqrt{\psi_{n 1}^{K}} e_{1}, \ldots, \sqrt{\psi_{n n}^{K}} e_{n}\right)_{n^{2} \times n}^{T} \text {, } \\
& V_{D}=\left(\sqrt{\eta_{11}} e_{1}, \ldots, \sqrt{\eta_{1 n}} e_{n}, \sqrt{\eta_{21}} e_{1}, \ldots, \sqrt{\eta_{2 n}} e_{n}, \ldots, \sqrt{\eta_{n 1}} e_{1}, \ldots, \sqrt{\eta_{n n}} e_{n}\right)_{n^{2} \times n}^{T} \text {. }
\end{aligned}
$$

We can get:

$$
\begin{array}{ll}
U_{A}^{R}\left(U_{A}^{R}\right)^{T}=\operatorname{diag}\left(\sum_{j=1}^{n} \phi_{1 j}^{R}, \ldots, \sum_{j=1}^{n} \phi_{n j}^{R}\right), & U_{A}^{I}\left(U_{A}^{I}\right)^{T}=\operatorname{diag}\left(\sum_{j=1}^{n} \phi_{1 j}^{I}, \ldots, \sum_{j=1}^{n} \phi_{n j}^{I}\right), \\
U_{A}^{J}\left(U_{A}^{J}\right)^{T}=\operatorname{diag}\left(\sum_{j=1}^{n} \phi_{1 j}^{J}, \ldots, \sum_{j=1}^{n} \phi_{n j}^{J}\right), & U_{A}^{K}\left(U_{A}^{K}\right)^{T}=\operatorname{diag}\left(\sum_{j=1}^{n} \phi_{1 j}^{K}, \ldots, \sum_{j=1}^{n} \phi_{n j}^{K}\right),
\end{array}
$$




$$
\begin{aligned}
& U_{B}^{R}\left(U_{B}^{R}\right)^{T}=\operatorname{diag}\left(\sum_{j=1}^{n} \varphi_{1 j}^{R}, \ldots, \sum_{j=1}^{n} \varphi_{n j}^{R}\right), \quad U_{B}^{I}\left(U_{B}^{I}\right)^{T}=\operatorname{diag}\left(\sum_{j=1}^{n} \varphi_{1 j}^{I}, \ldots, \sum_{j=1}^{n} \varphi_{n j}^{I}\right), \\
& U_{B}^{J}\left(U_{B}^{J}\right)^{T}=\operatorname{diag}\left(\sum_{j=1}^{n} \varphi_{1 j}^{J}, \ldots, \sum_{j=1}^{n} \varphi_{n j}^{J}\right), \quad U_{B}^{K}\left(U_{B}^{K}\right)^{T}=\operatorname{diag}\left(\sum_{j=1}^{n} \varphi_{1 j}^{K}, \ldots, \sum_{j=1}^{n} \varphi_{n j}^{K}\right), \\
& U_{C}^{R}\left(U_{C}^{R}\right)^{T}=\operatorname{diag}\left(\sum_{j=1}^{n} \psi_{1 j}^{R}, \ldots, \sum_{j=1}^{n} \psi_{n j}^{R}\right), \quad U_{C}^{I}\left(U_{C}^{I}\right)^{T}=\operatorname{diag}\left(\sum_{j=1}^{n} \psi_{1 j}^{I}, \ldots, \sum_{j=1}^{n} \psi_{n j}^{I}\right), \\
& U_{C}^{J}\left(U_{C}^{J}\right)^{T}=\operatorname{diag}\left(\sum_{j=1}^{n} \psi_{1 j}^{J}, \ldots, \sum_{j=1}^{n} \psi_{n j}^{J}\right), \quad U_{C}^{K}\left(U_{C}^{K}\right)^{T}=\operatorname{diag}\left(\sum_{j=1}^{n} \psi_{1 j}^{K}, \ldots, \sum_{j=1}^{n} \psi \psi_{n j}^{K}\right), \\
& U_{D}\left(U_{D}\right)^{T}=\operatorname{diag}\left(\sum_{j=1}^{n} \eta_{1 j}, \ldots, \sum_{j=1}^{n} \eta_{n j}\right), \quad\left(V_{A}^{R}\right)^{T} V_{A}^{R}=\operatorname{diag}\left(\sum_{j=1}^{n} \phi_{j 1}^{R}, \ldots, \sum_{j=1}^{n} \phi_{j n}^{R}\right), \\
& \left(V_{A}^{I}\right)^{T} V_{A}^{I}=\operatorname{diag}\left(\sum_{j=1}^{n} \phi_{j 1}^{I}, \ldots, \sum_{j=1}^{n} \phi_{j n}^{I}\right), \quad\left(V_{A}^{J}\right)^{T} V_{A}^{J}=\operatorname{diag}\left(\sum_{j=1}^{n} \phi_{j 1}^{J}, \ldots, \sum_{j=1}^{n} \phi_{j n}^{J}\right), \\
& \left(V_{A}^{K}\right)^{T} V_{A}^{K}=\operatorname{diag}\left(\sum_{j=1}^{n} \phi_{j 1}^{K}, \ldots, \sum_{j=1}^{n} \phi_{j n}^{K}\right), \quad\left(V_{B}^{R}\right)^{T} V_{B}^{R}=\operatorname{diag}\left(\sum_{j=1}^{n} \varphi_{j 1}^{R}, \ldots, \sum_{j=1}^{n} \varphi_{j n}^{R}\right), \\
& \left(V_{B}^{I}\right)^{T} V_{B}^{I}=\operatorname{diag}\left(\sum_{j=1}^{n} \varphi_{j 1}^{I}, \ldots, \sum_{j=1}^{n} \varphi_{j n}^{I}\right), \quad\left(V_{B}^{J}\right)^{T} V_{B}^{J}=\operatorname{diag}\left(\sum_{j=1}^{n} \varphi_{j 1}^{J}, \ldots, \sum_{j=1}^{n} \varphi_{j n}^{J}\right), \\
& \left(V_{B}^{K}\right)^{T} V_{B}^{K}=\operatorname{diag}\left(\sum_{j=1}^{n} \varphi_{j 1}^{K}, \ldots, \sum_{j=1}^{n} \varphi_{j n}^{K}\right), \quad\left(V_{C}^{R}\right)^{T} V_{C}^{R}=\operatorname{diag}\left(\sum_{j=1}^{n} \psi_{j 1}^{R}, \ldots, \sum_{j=1}^{n} \psi_{j n}^{R}\right), \\
& \left(V_{C}^{I}\right)^{T} V_{C}^{I}=\operatorname{diag}\left(\sum_{j=1}^{n} \psi_{j 1}^{I}, \ldots, \sum_{j=1}^{n} \psi_{j n}^{I}\right), \quad\left(V_{C}^{J}\right)^{T} V_{C}^{J}=\operatorname{diag}\left(\sum_{j=1}^{n} \psi_{j 1}^{J}, \ldots, \sum_{j=1}^{n} \psi_{j n}^{J}\right), \\
& \left(V_{C}^{K}\right)^{T} V_{C}^{K}=\operatorname{diag}\left(\sum_{j=1}^{n} \psi_{j 1}^{K}, \ldots, \sum_{j=1}^{n} \psi_{j n}^{K}\right), \quad\left(V_{D}\right)^{T} V_{D}=\operatorname{diag}\left(\sum_{j=1}^{n} \eta_{j 1}, \ldots, \sum_{j=1}^{n} \eta_{j n}\right) .
\end{aligned}
$$

Then, we can define:

$$
\begin{aligned}
& N_{A}^{R}=\operatorname{diag}\left(\sqrt{\sum_{j=1}^{n} \phi_{1}^{R}}, \ldots, \sqrt{\sum_{j=1}^{n} \phi_{n j}^{R}}\right), \quad N_{A}^{I}=\operatorname{diag}\left(\sqrt{\sum_{j=1}^{n} \phi_{1 j}^{I}}, \ldots, \sqrt{\sum_{j=1}^{n} \phi_{n j}^{I}}\right), \\
& N_{A}^{J}=\operatorname{diag}\left(\sqrt{\sum_{j=1}^{n} \phi_{1 j}^{J}}, \ldots, \sqrt{\sum_{j=1}^{n} \phi_{n j}^{J}}\right), \quad N_{A}^{K}=\operatorname{diag}\left(\sqrt{\sum_{j=1}^{n} \phi_{1 j}^{K}}, \ldots, \sqrt{\sum_{j=1}^{n} \phi_{n j}^{K}}\right),
\end{aligned}
$$

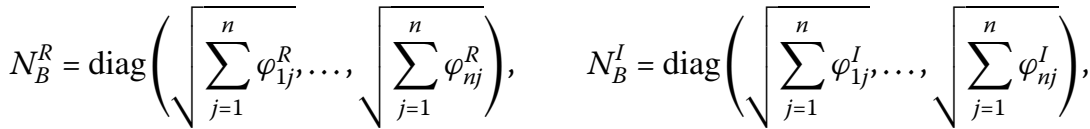

$$
\begin{aligned}
& N_{B}^{J}=\operatorname{diag}\left(\sqrt{\sum_{j=1}^{n} \varphi_{1 j}^{J}}, \ldots, \sqrt{\sum_{j=1}^{n} \varphi_{n j}^{J}}\right), \quad N_{B}^{K}=\operatorname{diag}\left(\sqrt{\sum_{j=1}^{n} \varphi_{1 j}^{K}}, \ldots, \sqrt{\sum_{j=1}^{n} \varphi_{n j}^{K}}\right), \\
& N_{C}^{R}=\operatorname{diag}\left(\sqrt{\sum_{j=1}^{n} \psi_{1 j}^{R}}, \ldots, \sqrt{\sum_{j=1}^{n} \psi_{n j}^{R}}\right), \quad N_{C}^{I}=\operatorname{diag}\left(\sqrt{\sum_{j=1}^{n} \psi_{1 j}^{I}}, \ldots, \sqrt{\sum_{j=1}^{n} \psi_{n j}^{I}}\right),
\end{aligned}
$$




$$
\begin{aligned}
& N_{C}^{J}=\operatorname{diag}\left(\sqrt{\sum_{j=1}^{n} \psi_{1 j}^{J}}, \ldots, \sqrt{\sum_{j=1}^{n} \psi_{n j}^{J}}\right), \quad N_{C}^{K}=\operatorname{diag}\left(\sqrt{\sum_{j=1}^{n} \psi_{1 j}^{K}}, \ldots, \sqrt{\sum_{j=1}^{n} \psi_{n j}^{K}}\right) \\
& N_{D}=\operatorname{diag}\left(\sqrt{\sum_{j=1}^{n} \eta_{1 j}}, \ldots, \sqrt{\sum_{j=1}^{n} \eta_{n j}}\right) .
\end{aligned}
$$

\subsection{Basic definitions and lemmas}

For the next work, we introduce the following definitions and lemmas.

Definition 1 A function $g(t) \in C\left((-\infty,+\infty), \mathbb{H}^{n}\right)$ is a solution of system (1) satisfying the initial value condition (2), if the following conditions are satisfied:

(i) $g(t)$ is absolutely continuous on each interval $\left(t_{k}, t_{k+1}\right) \subset(-\infty,+\infty), k=1,2, \ldots$,

(ii) for any $t_{k} \in[0,+\infty), k=1,2, \ldots, g\left(t_{k}^{+}\right)$and $\left(g\left(t_{k}^{-}\right)\right)$exist and $g\left(t_{k}^{+}\right)=g\left(t_{k}\right)$.

Lemma 1 ([28]) Let $\Upsilon^{*}=\left\{\Upsilon \in \mathbb{R}^{n^{2} \times n^{2}}: \Upsilon=\operatorname{diag}\left(\gamma_{11}, \ldots, \gamma_{1 n}, \ldots, \gamma_{n 1}, \ldots, \gamma_{n n}\right)\right.$, where $\left|\gamma_{i j}\right| \leq$ $1, i, j=1,2, \ldots, n\}$, then $\Upsilon^{T} \Upsilon \leq$ I. Furthermore, let

$$
\begin{aligned}
& \widetilde{D}=\left\{D=D_{0}+U_{D} \Upsilon_{D} V_{D}\right\}, \\
& \widetilde{C}=\left\{C=C_{0}+U_{C}^{R} \Upsilon_{C}^{R} V_{C}^{R}+\imath U_{C}^{I} \Upsilon_{C}^{I} V_{C}^{I}+\jmath U_{C}^{J} \Upsilon_{C}^{J} V_{C}^{J}+\kappa U_{C}^{K} \Upsilon_{C}^{K} V_{C}^{K}\right\}, \\
& \widetilde{B}=\left\{B=B_{0}+U_{B}^{R} \Upsilon_{B}^{R} V_{B}^{R}+\imath U_{B}^{I} \Upsilon_{B}^{I} V_{B}^{I}+\jmath U_{B}^{J} \Upsilon_{B}^{J} V_{B}^{J}+\kappa U_{B}^{K} \Upsilon_{B}^{K} V_{B}^{K}\right\}, \\
& \widetilde{A}=\left\{A=A_{0}+U_{A}^{R} \Upsilon_{A}^{R} V_{A}^{R}+\imath U_{A}^{I} \Upsilon_{A}^{I} V_{A}^{I}+\jmath U_{A}^{J} \Upsilon_{A}^{J} V_{A}^{J}+\kappa U_{A}^{K} \Upsilon_{A}^{K} V_{A}^{K}\right\} .
\end{aligned}
$$

Then for $\Upsilon_{C}, \Upsilon_{A}^{R}, \Upsilon_{A}^{I}, \Upsilon_{A}^{J}, \Upsilon_{A}^{K}, \Upsilon_{B}^{R}, \Upsilon_{B}^{I}, \Upsilon_{B}^{J}, \Upsilon_{B}^{K}, \Upsilon_{C}^{R}, \Upsilon_{C}^{I}, \Upsilon_{C}^{J}, \Upsilon_{C}^{K} \in \Upsilon^{*}, D_{I}=\widetilde{D}, C_{I}=\widetilde{C}, A_{I}=$ $\widetilde{A}, B_{I}=\widetilde{B}$.

Lemma 2 ([8]) If $U_{i}, V_{i}$ and $W_{i}(i=1,2, \ldots, m)$ are complex-valued matrices of appropriate dimension with $M$ satisfying $M^{*}=M$, then

$$
M+\sum_{i=1}^{m}\left(U_{i} V_{i} W_{i}+W_{i}^{*} V_{i}^{*} U_{i}^{*}\right)<0
$$

for all $V_{i}^{*} V_{i} \leq I(i=1,2, \ldots, m)$, if and only if there exist positive constants $\varepsilon_{i}(i=1,2, \ldots, m)$ such that

$$
M+\sum_{i=1}^{m}\left(\varepsilon_{i}^{-1} U_{i} U_{i}^{*}+\varepsilon_{i} W_{i}^{*} W_{i}\right)<0 .
$$

Lemma 3 ([13]) For a given Hermitian matrix,

$$
S=\left(\begin{array}{ll}
S_{11} & S_{12} \\
S_{21} & S_{22}
\end{array}\right)<0,
$$

where $S_{11}^{*}=S_{11}, S_{12}^{*}=S_{21}$, and $S_{22}^{*}=S_{22}$, is equivalent to the following conditions:

(i) $S_{22}<0$ and $S_{11}-S_{12} S_{22}^{-1} S_{21}<0$,

(ii) $S_{11}<0$ and $S_{22}-S_{21} S_{11}^{-1} S_{12}<0$. 
Lemma 4 ([35]) For any $a, b \in \mathbb{H}^{n}$, if $P \in \mathbb{H}^{n \times n}$ is a positive definite Hermitian matrix, then

$$
a^{*} b+b^{*} a \leq a^{*} P a+b^{*} P^{-1} b .
$$

Lemma 5 ([35]) For any positive definite constant Hermitian matrix $W \in \mathbb{H}^{n \times n}$ and any scalar function $\omega(s):[a, b] \rightarrow \mathbb{H}^{n}$ with scalars $a<b$ such that the integrals concerned are well defined,

$$
\left(\int_{a}^{b} \omega(s) d s\right)^{*} W\left(\int_{a}^{b} \omega(s) d s\right) \leq(b-a) \int_{a}^{b} \omega^{*}(s) W \omega(s) d s .
$$

Lemma 6 ([35]) If $G(q): \mathbb{H}^{n} \rightarrow \mathbb{H}^{n}$ is a continuous map and satisfies the following conditions:

(i) $G(q)$ is injective on $\mathbb{H}^{n}$,

(ii) $\lim _{\|q\| \rightarrow \infty}\|G(q)\|=\infty$,

then $G(q)$ is a homeomorphism of $\mathbb{H}^{n}$ onto itself.

Lemma 7 ([37]) Let $A=A_{1}+A_{2} J$ and $B=B_{1}+B_{2} J$, where $A_{1}, A_{2}, B_{1}, B_{2} \in \mathbb{C}^{n \times n}$ and $A, B \in$ $\mathbb{H}^{n \times n}$. Then

(1) $A^{*}=A_{1}^{*}-A_{2} J^{T}$;

(2) $A B=\left(A_{1} B_{1}-A_{2} \bar{B}_{2}\right)+\left(A_{1} B_{2}+A_{2} \bar{B}_{1}\right) \mathrm{J}$

where $\bar{B}_{1}$ and $\bar{B}_{2}$ denote the conjugate matrices of $B_{1}$ and $B_{2}$, respectively.

Lemma 8 ([37]) Let $Q \in \mathbb{H}^{n \times n}$ be a Hermite matrix, $Q=Q_{1}+Q_{2}$ J where $Q_{1}, Q_{2} \in \mathbb{C}^{n \times n}$ and $Q \in \mathbb{H}^{n \times n}$. Then $Q<0$ is equivalent to

$$
\left(\begin{array}{cc}
Q_{1} & -Q_{2} \\
\bar{Q}_{2} & \bar{Q}_{1}
\end{array}\right)<0,
$$

where $\bar{Q}_{1}$ and $\bar{Q}_{2}$ denote the conjugate matrices of $Q_{1}$ and $Q_{2}$, respectively.

\section{Main results}

In this section, we study the existence and uniqueness of an equilibrium point of system (1) and analyze the global robust stability of the unique equilibrium point for system (1) under assumptions $\left(\mathbf{A}_{1}\right),\left(\mathbf{A}_{2}\right)$, and $\left(\mathbf{A}_{3}\right)$.

Theorem 1 Under assumptions $\left(\mathbf{A}_{1}\right),\left(\mathbf{A}_{2}\right)$, and $\left(\mathbf{A}_{3}\right), Q V N N(1)$ has a unique equilibrium point, which is globally robust stable, if there are a positive definite Hermitian matrix $P_{1}$, four positive diagonal matrices $P_{i}(i=2,3,4)$ and $R$, and positive constants $\varepsilon_{i}$ $(i=1,2, \ldots, 34)$ such that the following linear matrix inequality holds:

$$
\Xi=\left(\Xi_{i j}\right)_{41 \times 41}<0,
$$

where

$$
\begin{aligned}
\Xi_{11}= & -P_{1} D_{0}-D_{0}^{T} P_{1}+P_{2}+\delta^{2} P_{3}+L\left(2 P_{2}+R\right) L+\varepsilon_{1}\left(V_{D}\right)^{T} V_{D} \\
& +\varepsilon_{15}\left(V_{D}\right)^{T} V_{D}, \quad \Xi_{12}=\frac{3}{2} P_{1} A_{0}, \quad \Xi_{13}=\frac{3}{2} P_{1} B_{0},
\end{aligned}
$$




$$
\begin{aligned}
& \Xi_{14}=\frac{3}{2} P_{1} C_{0}, \quad \Xi_{15}=\left(D_{0}\right)^{T} P_{1} D_{0}, \quad \Xi_{16}=-\frac{1}{2} P_{1} D_{0}, \quad \Xi_{18}=P_{1} N_{D}, \\
& \Xi_{19}=\frac{3}{2} P_{1} N_{A}^{R}, \quad \Xi_{1,10}=\frac{3}{2} P_{1} N_{A}^{I}, \quad \Xi_{1.11}=\frac{3}{2} P_{1} N_{A}^{J}, \quad \Xi_{1.12}=\frac{3}{2} P_{1} N_{A}^{K}, \\
& \Xi_{1.13}=\frac{3}{2} P_{1} N_{B}^{R}, \quad \Xi_{1.14}=\frac{3}{2} P_{1} N_{B}^{I}, \quad \Xi_{1.15}=\frac{3}{2} P_{1} N_{B}^{J}, \quad \Xi_{1.16}=\frac{3}{2} P_{1} N_{B}^{K}, \\
& \Xi_{1.17}=\frac{3}{2} P_{1} N_{C}^{R}, \quad \Xi_{1.18}=\frac{3}{2} P_{1} N_{C}^{I}, \quad \Xi_{1.19}=\frac{3}{2} P_{1} N_{C}^{J}, \quad \Xi_{1.20}=\frac{3}{2} P_{1} N_{C}^{K}, \\
& \Xi_{1.21}=D_{0}^{T} P_{1} N_{D}, \quad \Xi_{1.23}=\left(U_{D} \Upsilon_{D} V_{D}\right)^{T} P_{1} N_{D}, \\
& \Xi_{1.24}=\frac{1}{2} P_{1} N_{D}, \quad \Xi_{21}=\frac{3}{2}\left(A_{0}\right)^{*} P_{1}, \\
& \Xi_{22}=P_{4}-2 P_{2}+\varepsilon_{2}\left(V_{A}^{R}\right)^{T} V_{A}^{R}+\varepsilon_{3}\left(V_{A}^{I}\right)^{T} V_{A}^{I}+\varepsilon_{4}\left(V_{A}^{J}\right)^{T} V_{A}^{J}+\varepsilon_{5}\left(V_{A}^{K}\right)^{T} V_{A}^{K} \\
& +\varepsilon_{18}\left(V_{A}^{R}\right)^{T} V_{A}^{R}+\varepsilon_{19}\left(V_{A}^{I}\right)^{T} V_{A}^{I}+\varepsilon_{20}\left(V_{A}^{J}\right)^{T} V_{A}^{J}+\varepsilon_{21}\left(V_{A}^{K}\right)^{T} V_{A}^{K}, \\
& \Xi_{27}=\left(A_{0}\right)^{*} P_{1}, \quad \Xi_{31}=\frac{3}{2}\left(B_{0}\right)^{*} P_{1}, \\
& \Xi_{33}=-P_{4}+\varepsilon_{6}\left(V_{B}^{R}\right)^{T} V_{B}^{R}+\varepsilon_{7}\left(V_{B}^{I}\right)^{T} V_{B}^{I}+\varepsilon_{8}\left(V_{B}^{J}\right)^{T} V_{B}^{J}+\varepsilon_{9}\left(V_{B}^{K}\right)^{T} V_{B}^{K} \\
& +\varepsilon_{22}\left(V_{B}^{R}\right)^{T} V_{B}^{R}+\varepsilon_{23}\left(V_{B}^{I}\right)^{T} V_{B}^{I}+\varepsilon_{24}\left(V_{B}^{J}\right)^{T} V_{B}^{J}+\varepsilon_{25}\left(V_{B}^{K}\right)^{T} V_{B}^{K}, \\
& \Xi_{37}=\left(B_{0}\right)^{*} P_{1}, \quad \Xi_{41}=\frac{3}{2}\left(C_{0}\right)^{*} P_{1}, \\
& \Xi_{44}=-R+\varepsilon_{10}\left(V_{C}^{R}\right)^{T} V_{C}^{R}+\varepsilon_{11}\left(V_{C}^{I}\right)^{T} V_{C}^{I}+\varepsilon_{12}\left(V_{C}^{J}\right)^{T} V_{C}^{J}+\varepsilon_{13}\left(V_{C}^{K}\right)^{T} V_{C}^{K} \\
& +\varepsilon_{26}\left(V_{C}^{R}\right)^{T} V_{C}^{R}+\varepsilon_{27}\left(V_{C}^{I}\right)^{T} V_{C}^{I}+\varepsilon_{28}\left(V_{C}^{J}\right)^{T} V_{C}^{J}+\varepsilon_{29}\left(V_{C}^{K}\right)^{T} V_{C}^{K}, \\
& \Xi_{47}=\left(C_{0}\right)^{*} P_{1}, \quad \Xi_{51}=\left(D_{0}\right)^{T} P_{1} D_{0}, \\
& \Xi_{55}=-P_{3}+\varepsilon_{14}\left(V_{D}\right)^{T} V_{D}+\varepsilon_{16}\left(V_{D}\right)^{T} V_{D}+\varepsilon_{33}\left(V_{D}\right)^{T} V_{D}, \\
& \Xi_{56}=-\left(D_{0}\right)^{T} P_{1} D_{0}, \quad \Xi_{57}=-\left(D_{0}\right)^{T} P_{1}, \quad \Xi_{5.22}=D_{0}^{T} P_{1} N_{D}, \quad \Xi_{5.37}=P_{1} N_{D}, \\
& \Xi_{5.39}=D_{0}^{T} P_{1} N_{D}, \quad \Xi_{5.41}=\left(U_{D} \Upsilon_{D} V_{D}\right)^{T} P_{1} N_{D}, \quad \Xi_{61}=-\frac{1}{2} D_{0}^{T} P_{1}, \\
& \Xi_{65}=-\left(D_{0}\right)^{T} P_{1} D_{0}, \quad \Xi_{66}=-P_{2}+\varepsilon_{17}\left(V_{D}\right)^{T} V_{D}+\varepsilon_{32}\left(V_{D}\right)^{T} V_{D}+\varepsilon_{34}\left(V_{D}\right)^{T} V_{D}, \\
& \Xi_{67}=-\left(D_{0}\right)^{T} P_{1}, \quad \Xi_{6.38}=P_{1} N_{D}, \quad \Xi_{6.40}=D_{0}^{T} P_{1} N_{D}, \\
& \Xi_{72}=P_{1} A_{0}, \quad \Xi_{73}=P_{1} B_{0}, \quad \Xi_{74}=P_{1} C_{0}, \quad \Xi_{75}=-P_{1} D_{0}, \quad \Xi_{76}=-P_{1} D_{0}, \\
& \Xi_{77}=-P_{1}-P_{1}+\varepsilon_{30}\left(V_{D}\right)^{T} V_{D}+\varepsilon_{31}\left(V_{D}\right)^{T} V_{D}, \\
& \Xi_{7.25}=P_{1} N_{A}^{R}, \quad \Xi_{7.26}=P_{1} N_{A}^{I}, \quad \Xi_{7.27}=P_{1} N_{A}^{J}, \quad \Xi_{7.28}=P_{1} N_{A}^{K}, \\
& \Xi_{7.29}=P_{1} N_{B}^{R}, \quad \Xi_{7.30}=P_{1} N_{B}^{I}, \quad \Xi_{7.31}=P_{1} N_{B}^{J}, \quad \Xi_{7.32}=P_{1} N_{B}^{K}, \\
& \Xi_{7.33}=P_{1} N_{C}^{R}, \quad \Xi_{7.34}=P_{1} N_{C}^{I}, \quad \Xi_{7.35}=P_{1} N_{C}^{J}, \quad \Xi_{7.36}=P_{1} N_{C}^{K}, \\
& \Xi_{81}=\left(N_{D}\right)^{T} P_{1}, \quad \Xi_{88}=-\varepsilon_{1} I, \quad \Xi_{91}=\frac{3}{2}\left(N_{A}^{R}\right)^{T} P_{1}, \\
& \Xi_{99}=-\varepsilon_{2} I, \quad \Xi_{10.1}=\frac{3}{2}\left(N_{A}^{I}\right)^{T} P_{1}, \quad \Xi_{10.10}=-\varepsilon_{3} I, \quad \Xi_{11.1}=\frac{3}{2}\left(N_{A}^{J}\right)^{T} P_{1}, \\
& \Xi_{11.11}=-\varepsilon_{4} I, \quad \Xi_{12.1}=\frac{3}{2}\left(N_{A}^{K}\right)^{T} P_{1}, \quad \Xi_{12.12}=-\varepsilon_{5} I, \quad \Xi_{13.1}=\frac{3}{2}\left(N_{B}^{R}\right)^{T} P_{1},
\end{aligned}
$$




$$
\begin{array}{llll}
\Xi_{13.13}=-\varepsilon_{6} I, & \Xi_{14.1}=\frac{3}{2}\left(N_{B}^{I}\right)^{T} P_{1}, & \Xi_{14.14}=-\varepsilon_{7} I, & \Xi_{15.1}=\frac{3}{2}\left(N_{B}^{J}\right)^{T} P_{1}, \\
\Xi_{15.15}=-\varepsilon_{8} I, & \Xi_{16.1}=\frac{3}{2}\left(N_{B}^{K}\right)^{T} P_{1}, & \Xi_{16.16}=-\varepsilon_{9} I, & \Xi_{17.1}=\frac{3}{2}\left(N_{C}^{R}\right)^{T} P_{1}, \\
\Xi_{17.17}=-\varepsilon_{10} I, & \Xi_{18.1}=\frac{3}{2}\left(N_{C}^{I}\right)^{T} P_{1}, & \Xi_{18.18}=-\varepsilon_{11} I, & \Xi_{19.1}=\frac{3}{2}\left(N_{C}^{J}\right)^{T} P_{1}, \\
\Xi_{19.19}=-\varepsilon_{12} I, & \Xi_{20.1}=\frac{3}{2}\left(N_{C}^{K}\right)^{T} P_{1}, & \Xi_{20.20}=-\varepsilon_{13} I, & \Xi_{21.1}=\left(N_{D}\right)^{T} P_{1} D_{0}, \\
\Xi_{21.21}=-\varepsilon_{14} I, & \Xi_{22.5}=\left(N_{D}\right)^{T} P_{1} D_{0}, & \Xi_{22.22}=-\varepsilon_{15} I, & \\
\Xi_{23.1}=\left(N_{D}\right)^{T} P_{1} U_{D} \Upsilon_{D} V_{D}, \quad \Xi_{23.23}=-\varepsilon_{16} I, & \Xi_{24.1}=\frac{1}{2}\left(N_{D}\right)^{T} P_{1}, \\
\Xi_{24.24}=-\varepsilon_{17} I, & \Xi_{25.7}=\left(N_{A}^{R}\right)^{T} P_{1}, & \Xi_{25.25}=-\varepsilon_{18} I, & \Xi_{26.7}=\left(N_{A}^{I}\right)^{T} P_{1}, \\
\Xi_{26.26}=-\varepsilon_{19} I, & \Xi_{27.7}=\left(N_{A}^{J}\right)^{T} P_{1}, & \Xi_{27.27}=-\varepsilon_{20} I, & \Xi_{28.7}=\left(N_{A}^{K}\right)^{T} P_{1}, \\
\Xi_{28.28}=-\varepsilon_{21} I, & \Xi_{29.7}=\left(N_{B}^{R}\right)^{T} P_{1}, & \Xi_{29.29}=-\varepsilon_{22} I, & \Xi_{30.7}=\left(N_{B}^{I}\right)^{T} P_{1}, \\
\Xi_{30.30}=-\varepsilon_{23} I, & \Xi_{31.7}=\left(N_{B}^{J}\right)^{T} P_{1}, & \Xi_{31.31}=-\varepsilon_{24} I, & \Xi_{32.7}=\left(N_{B}^{K}\right)^{T} P_{1}, \\
\Xi_{32.32}=-\varepsilon_{25} I, & \Xi_{33.7}=\left(N_{C}^{R}\right)^{T} P_{1}, & \Xi_{33.33}=-\varepsilon_{26} I, & \Xi_{347}=\left(N_{C}^{I}\right)^{T} P_{1}, \\
\Xi_{34.34}=-\varepsilon_{27} I, & \Xi_{35.7}=\left(N_{C}^{J}\right)^{T} P_{1}, & \Xi_{35.35}=-\varepsilon_{28} I, & \Xi_{36.7}=\left(N_{C}^{K}\right)^{T} P_{1}, \\
\Xi_{36.36}=-\varepsilon_{29} I, & \Xi_{37.5}=\left(N_{D}\right)^{T} P_{1} \quad \Xi_{37.37}=-\varepsilon_{30} I, \quad & \Xi_{38.6}=\left(N_{D}\right)^{T} P_{1}, \\
\Xi_{38.38}=-\varepsilon_{31} I, & \Xi_{39.5}=\left(N_{D}\right)^{T} P_{1} D_{0}, & \Xi_{39.39}=-\varepsilon_{32} I, & \Xi_{40.6}=\left(N_{D}\right)^{T} P_{1} D_{0}, \\
\Xi_{40.40}=-\varepsilon_{33} I, & \Xi_{41.5}=\left(N_{D}\right)^{T} P_{1} U_{D} \Upsilon_{D} V_{D}, & \Xi_{41.41}=-\varepsilon_{34} I,
\end{array}
$$

and the other entries in $\Xi$ are zero.

Proof We prove this theorem in three steps:

Step 1: Matrices $\Xi$ and $M$ are equivalent in negative definiteness.

$$
\mathrm{M}=\left(\begin{array}{ccccccc}
M_{11} & \frac{3}{2} P_{1} A & \frac{3}{2} P_{1} B & \frac{3}{2} P_{1} C & D^{T} P_{1} D & -\frac{1}{2} P_{1} D & 0 \\
\star & P_{4}-2 P_{2} & 0 & 0 & 0 & 0 & A^{*} P_{1} \\
\star & \star & -P_{4} & 0 & 0 & 0 & B^{*} P_{1} \\
\star & \star & \star & -R & 0 & 0 & C^{*} P_{1} \\
\star & \star & \star & \star & -P_{3} & -D^{T} P_{1} D & -D^{T} P_{1} \\
\star & \star & \star & \star & \star & -P_{2} & -D^{T} P_{1} \\
\star & \star & \star & \star & \star & \star & -P_{1}-P_{1}
\end{array}\right),
$$

where $M_{11}=-P_{1} D-D^{T} P_{1}+\delta^{2} P_{3}+P_{2}+L\left(2 P_{2}+R\right) L$.

From Lemma 1 , for any $D \in D_{I}, C \in C_{I}, A \in A_{I}, B \in B_{I}$, we can obtain that

$$
\begin{aligned}
& D=D_{0}+U_{d} \Upsilon_{d} V_{d}, \\
& A=A_{0}+U_{A}^{R} \Upsilon_{A}^{R} V_{A}^{R}+\imath U_{A}^{I} \Upsilon_{A}^{I} V_{A}^{I}+{ }_{\jmath} U_{A}^{J} \Upsilon_{A}^{J} V_{A}^{J}+\kappa U_{A}^{K} \Upsilon_{A}^{K} V_{A}^{K}, \\
& B=B_{0}+U_{B}^{R} \Upsilon_{B}^{R} V_{B}^{R}+\imath U_{B}^{I} \Upsilon_{B}^{I} V_{B}^{I}+\jmath U_{B}^{J} \Upsilon_{B}^{J} V_{B}^{J}+\kappa U_{B}^{K} \Upsilon_{B}^{K} V_{B}^{K}, \\
& C=C_{0}+U_{C}^{R} \Upsilon_{C}^{R} V_{C}^{R}+\imath U_{C}^{I} \Upsilon_{C}^{I} V_{C}^{I}+{ }_{\jmath} U_{C}^{J} \Upsilon_{C}^{J} V_{C}^{J}+\kappa U_{C}^{K} \Upsilon_{C}^{K} V_{C}^{K},
\end{aligned}
$$


and for $\left(\Upsilon_{D}\right),\left(\Upsilon_{A}^{X}\right),\left(\Upsilon_{B}^{X}\right),\left(\Upsilon_{C}^{X}\right) \in \Upsilon^{*}$, where $X$ denotes $R, I, J, K$, respectively. We have $\left(\Upsilon_{A}^{X}\right)^{T} \Upsilon_{A}^{X} \leq I,\left(\Upsilon_{B}^{X}\right)^{T} \Upsilon_{B}^{X} \leq I,\left(\Upsilon_{C}^{X}\right)^{T} \Upsilon^{X} \leq I,\left(\Upsilon_{D}\right)^{T} \Upsilon_{D} \leq I$.

By substituting (5), (6), (7), and (8) into (4), we can get

$$
\begin{aligned}
& \mathrm{M}=\left(\begin{array}{ccccccc}
M_{11} & \frac{3}{2} P_{1} A_{0} & \frac{3}{2} P_{1} B_{0} & \frac{3}{2} P_{1} C_{0} & D_{0}^{T} P_{1} D_{0} & -\frac{1}{2} P_{1} D_{0} & 0 \\
\star & P_{4}-2 P_{2} & 0 & 0 & 0 & 0 & \left(A_{0}\right)^{*} P_{1} \\
\star & \star & -P_{4} & 0 & 0 & 0 & \left(B_{0}\right)^{*} P_{1} \\
\star & \star & \star & -R & 0 & 0 & \left(C_{0}\right)^{*} P_{1} \\
\star & \star & \star & \star & -P_{3} & -D_{0}^{T} P_{1} D_{0} & -D_{0}^{T} P_{1} \\
\star & \star & \star & \star & \star & -P_{2} & -D_{0}^{T} P_{1} \\
\star & \star & \star & \star & \star & \star & -P_{1}-P_{1}
\end{array}\right) \\
& +\left(\left(-U_{D}^{T} P_{1}\right) \lambda_{1}\right)^{T} \Upsilon_{D}\left(\left(V_{D}\right) \lambda_{1}\right)+\left(\left(V_{D}\right) \lambda_{1}\right)^{T}\left(\Upsilon_{D}\right)^{T}\left(\left(-U_{D}^{T} P_{1}\right) \lambda_{1}\right) \\
& \left.+\frac{3}{2}\left(\left(\left(U_{A}^{R}\right)^{T} P_{1}\right) \lambda_{1}\right)^{T}\left(\Upsilon_{A}^{R}\right)\left(\left(V_{A}^{R}\right) \lambda_{2}\right)+\frac{3}{2}\left(\left(V_{A}^{R}\right) \lambda_{2}\right)^{T}\left(\Upsilon_{A}^{R}\right)^{T}\left(\left(U_{A}^{R}\right)^{T} P_{1}\right) \lambda_{1}\right) \\
& +\frac{3}{2}\left(\left(\left(i U_{A}^{I}\right)^{*} P_{1}\right) \lambda_{1}\right)^{*}\left(\Upsilon_{A}^{I}\right)\left(\left(V_{A}^{I}\right) \lambda_{2}\right)+\frac{3}{2}\left(\left(V_{A}^{I}\right) \lambda_{2}\right)^{T}\left(\Upsilon_{A}^{R}\right)^{T}\left(\left(\left(i U_{A}^{I}\right)^{*} P_{1}\right) \lambda_{1}\right) \\
& +\frac{3}{2}\left(\left(\left(j U_{A}^{J}\right)^{*} P_{1}\right) \lambda_{1}\right)^{*}\left(\Upsilon_{A}^{J}\right)\left(\left(V_{A}^{J}\right) \lambda_{2}\right)+\frac{3}{2}\left(\left(V_{A}^{J}\right) \lambda_{2}\right)^{T}\left(\Upsilon_{A}^{J}\right)^{T}\left(\left(\left(j U_{A}^{J}\right)^{*} P_{1}\right) \lambda_{1}\right) \\
& +\frac{3}{2}\left(\left(\left(k U_{A}^{K}\right)^{*} P_{1}\right) \lambda_{1}\right)^{*}\left(\Upsilon_{A}^{K}\right)\left(\left(V_{A}^{K}\right) \lambda_{2}\right)+\frac{3}{2}\left(\left(V_{A}^{K}\right) \lambda_{2}\right)^{T}\left(\Upsilon_{A}^{K}\right)^{T}\left(\left(\left(k U_{A}^{K}\right)^{*} P_{1}\right) \lambda_{1}\right) \\
& +\frac{3}{2}\left(\left(\left(U_{B}^{R}\right)^{T} P_{1}\right) \lambda_{1}\right)^{T}\left(\Upsilon_{A}^{R}\right)\left(\left(V_{B}^{R}\right) \lambda_{3}\right)+\frac{3}{2}\left(\left(V_{B}^{R}\right) \lambda_{3}\right)^{T}\left(\Upsilon_{B}^{R}\right)^{T}\left(\left(\left(U_{B}^{R}\right)^{T} P_{1}\right) \lambda_{1}\right) \\
& +\frac{3}{2}\left(\left(\left(i U_{B}^{I}\right)^{*} P_{1}\right) \lambda_{1}\right)^{*}\left(\Upsilon_{B}^{I}\right)\left(\left(V_{B}^{I}\right) \lambda_{3}\right)+\frac{3}{2}\left(\left(V_{B}^{I}\right) \lambda_{3}\right)^{T}\left(\Upsilon_{B}^{I}\right)^{T}\left(\left(\left(i U_{B}^{I}\right)^{*} P_{1}\right) \lambda_{1}\right) \\
& +\frac{3}{2}\left(\left(\left(j U_{B}^{J}\right)^{*} P_{1}\right) \lambda_{1}\right)^{*}\left(\Upsilon_{B}^{J}\right)\left(\left(V_{B}^{J}\right) \lambda_{3}\right)+\frac{3}{2}\left(\left(V_{B}^{J}\right) \lambda_{3}\right)^{T}\left(\Upsilon_{B}^{J}\right)^{T}\left(\left(\left(j U_{B}^{J}\right)^{*} P_{1}\right) \lambda_{1}\right) \\
& +\frac{3}{2}\left(\left(\left(k U_{B}^{K}\right)^{*} P_{1}\right) \lambda_{1}\right)^{*}\left(\Upsilon_{B}^{K}\right)\left(\left(V_{B}^{K}\right) \lambda_{3}\right)+\frac{3}{2}\left(\left(V_{B}^{K}\right) \lambda_{3}\right)^{T}\left(\Upsilon_{B}^{K}\right)^{T}\left(\left(\left(k U_{B}^{K}\right)^{*} P_{1}\right) \lambda_{1}\right) \\
& +\frac{3}{2}\left(\left(\left(U_{C}^{R}\right)^{T} P_{1}\right) \lambda_{1}\right)^{T}\left(\Upsilon_{C}^{R}\right)\left(\left(V_{C}^{R}\right) \lambda_{4}\right)+\frac{3}{2}\left(\left(V_{C}^{R}\right) \lambda_{4}\right)^{T}\left(\Upsilon_{C}^{R}\right)^{T}\left(\left(\left(U_{C}^{R}\right)^{T} P_{1}\right) \lambda_{1}\right) \\
& +\frac{3}{2}\left(\left(\left(i U_{C}^{I}\right)^{*} P_{1}\right) \lambda_{1}\right)^{*}\left(\Upsilon_{C}^{I}\right)\left(\left(V_{C}^{I}\right) \lambda_{4}\right)+\frac{3}{2}\left(\left(V_{C}^{I}\right) \lambda_{4}\right)^{T}\left(\Upsilon_{C}^{I}\right)^{T}\left(\left(\left(i U_{C}^{I}\right)^{*} P_{1}\right) \lambda_{1}\right) \\
& +\frac{3}{2}\left(\left(\left(j U_{C}^{J}\right)^{*} P_{1}\right) \lambda_{1}\right)^{*}\left(\Upsilon_{C}^{J}\right)\left(\left(V_{C}^{J}\right) \lambda_{4}\right)+\frac{3}{2}\left(\left(V_{C}^{J}\right) \lambda_{4}\right)^{T}\left(\Upsilon_{C}^{J}\right)^{T}\left(\left(\left(j U_{C}^{J}\right)^{*} P_{1}\right) \lambda_{1}\right) \\
& +\frac{3}{2}\left(\left(\left(k U_{C}^{K}\right)^{*} P_{1}\right) \lambda_{1}\right)^{*}\left(\Upsilon_{C}^{K}\right)\left(V_{C}^{K} \lambda_{4}\right)+\frac{3}{2}\left(\left(V_{C}^{K}\right) \lambda_{4}\right)^{T}\left(\Upsilon_{C}^{K}\right)^{T}\left(\left(\left(k U_{C}^{K}\right)^{*} P_{1}\right) \lambda_{1}\right) \\
& +\left(\left(U_{D}^{T} P_{1} D_{0}\right) \lambda_{1}\right)^{T} \Upsilon_{D}\left(\left(V_{D}\right) \lambda_{5}\right)+\left(\left(V_{D}\right) \lambda_{5}\right)^{T} \Upsilon_{D}^{T}\left(\left(U_{D}^{T} P_{1} D_{0}\right) \lambda_{1}\right) \\
& +\left(\left(U_{D}^{T} P_{1} D_{0}\right) \lambda_{5}\right)^{T} \Upsilon_{D}\left(\left(V_{D}\right) \lambda_{1}\right)+\left(\left(V_{D}\right) \lambda_{1}\right)^{T} \Upsilon_{D}^{T}\left(\left(U_{D}^{T} P_{1} D_{0}\right) \lambda_{5}\right) \\
& +\left(\left(U_{D}^{T} P_{1} U_{D} \Upsilon_{D} V_{D}\right) \lambda_{1}\right)^{T} \Upsilon_{D}\left(\left(V_{D}\right) \lambda_{5}\right)+\left(\left(V_{D}\right) \lambda_{5}\right)^{T} \Upsilon_{D}^{T}\left(\left(U_{D}^{T} P_{1} U_{D} \Upsilon_{D} V_{D}\right) \lambda_{1}\right) \\
& +\frac{1}{2}\left(\left(-U_{D}^{T} P_{1}\right) \lambda_{1}\right)^{T} \Upsilon_{D}\left(\left(V_{D}\right) \lambda_{6}\right)+\frac{1}{2}\left(\left(V_{D}\right) \lambda_{6}\right)^{T} \Upsilon_{D}^{T}\left(\left(-U_{D}^{T} P_{1}\right) \lambda_{1}\right) \\
& +\left(\left(\left(U_{A}^{R}\right)^{T} P_{1}\right) \lambda_{7}\right)^{T}\left(\Upsilon_{A}^{R}\right)\left(\left(V_{A}^{R}\right) \lambda_{2}\right)+\left(\left(V_{A}^{R}\right) \lambda_{2}\right)^{T}\left(\Upsilon_{A}^{R}\right)^{T}\left(\left(\left(U_{A}^{R}\right)^{T} P_{1}\right) \lambda_{7}\right) \\
& +\left(\left(\left(i U_{A}^{I}\right)^{*} P_{1}\right) \lambda_{7}\right)^{*}\left(\Upsilon_{A}^{I}\right)\left(\left(V_{A}^{I}\right) \lambda_{2}\right)+\left(\left(V_{A}^{I}\right) \lambda_{2}\right)^{T}\left(\Upsilon_{A}^{I}\right)^{T}\left(\left(\left(i U_{A}^{I}\right)^{*} P_{1}\right) \lambda_{7}\right) \\
& +\left(\left(\left(j U_{A}^{J}\right)^{*} P_{1}\right) \lambda_{7}\right)^{*}\left(\Upsilon_{A}^{J}\right)\left(\left(V_{A}^{J}\right) \lambda_{2}\right)+\left(\left(V_{A}^{J}\right) \lambda_{2}\right)^{T}\left(\Upsilon_{A}^{J}\right)^{T}\left(\left(\left(j U_{A}^{J}\right)^{*} P_{1}\right) \lambda_{7}\right)
\end{aligned}
$$




$$
\begin{aligned}
& +\left(\left(\left(k U_{A}^{K}\right)^{*} P_{1}\right) \lambda_{7}\right)^{*}\left(\Upsilon_{A}^{K}\right)\left(\left(V_{A}^{K}\right) \lambda_{2}\right)+\left(\left(V_{A}^{K}\right) \lambda_{2}\right)^{T}\left(\Upsilon_{A}^{K}\right)^{T}\left(\left(\left(k U_{A}^{K}\right)^{*} P_{1}\right) \lambda_{7}\right) \\
& +\left(\left(\left(U_{B}^{R}\right)^{T} P_{1}\right) \lambda_{7}\right)^{T}\left(\Upsilon_{B}^{R}\right)\left(\left(V_{B}^{R}\right) \lambda_{3}\right)+\left(\left(V_{B}^{R}\right) \lambda_{3}\right)^{T}\left(\Upsilon_{B}^{R}\right)^{T}\left(\left(\left(U_{B}^{R}\right)^{T} P_{1}\right) \lambda_{7}\right) \\
& +\left(\left(\left(i U_{B}^{I}\right)^{*} P_{1}\right) \lambda_{7}\right)^{*}\left(\Upsilon_{B}^{I}\right)\left(\left(V_{B}^{I}\right) \lambda_{3}\right)+\left(\left(V_{B}^{I}\right) \lambda_{3}\right)^{T}\left(\Upsilon_{B}^{I}\right)^{T}\left(\left(\left(i U_{B}^{I}\right)^{*} P_{1}\right) \lambda_{7}\right) \\
& +\left(\left(\left(j U_{B}^{J}\right)^{*} P_{1}\right) \lambda_{7}\right)^{*}\left(\Upsilon_{B}^{J}\right)\left(\left(V_{B}^{J}\right) \lambda_{3}\right)+\left(\left(V_{B}^{J}\right) \lambda_{3}\right)^{T}\left(\Upsilon_{B}^{J}\right)^{T}\left(\left(\left(j U_{B}^{J}\right)^{*} P_{1}\right) \lambda_{7}\right) \\
& +\left(\left(\left(k U_{B}^{K}\right)^{*} P_{1}\right) \lambda_{7}\right)^{*}\left(\Upsilon_{B}^{K}\right)\left(\left(V_{B}^{K}\right) \lambda_{3}\right)+\left(\left(V_{B}^{K}\right) \lambda_{3}\right)^{T}\left(\Upsilon_{B}^{K}\right)^{T}\left(\left(\left(k U_{B}^{K}\right)^{*} P_{1}\right) \lambda_{7}\right) \\
& +\left(\left(\left(U_{C}^{R}\right)^{T} P_{1}\right) \lambda_{7}\right)^{T}\left(\Upsilon_{C}^{R}\right)\left(\left(V_{C}^{R}\right) \lambda_{4}\right)+\left(\left(V_{C}^{R}\right) \lambda_{4}\right)^{T}\left(\Upsilon_{C}^{R}\right)^{T}\left(\left(\left(U_{C}^{R}\right)^{T} P_{1}\right) \lambda_{7}\right) \\
& +\left(\left(\left(i U_{C}^{I}\right)^{*} P_{1}\right) \lambda_{7}\right)^{*}\left(\Upsilon_{C}^{I}\right)\left(\left(V_{C}^{I}\right) \lambda_{4}\right)+\left(\left(V_{C}^{I}\right) \lambda_{4}\right)^{T}\left(\Upsilon_{C}^{I}\right)^{T}\left(\left(\left(i U_{C}^{I}\right)^{*} P_{1}\right) \lambda_{7}\right) \\
& +\left(\left(\left(j U_{C}^{J}\right)^{*} P_{1}\right) \lambda_{7}\right)^{*}\left(\Upsilon_{C}^{J}\right)\left(\left(V_{C}^{J}\right) \lambda_{4}\right)+\left(\left(V_{C}^{J}\right) \lambda_{4}\right)^{T}\left(\Upsilon_{C}^{J}\right)^{T}\left(\left(\left(j U_{C}^{J}\right)^{*} P_{1}\right) \lambda_{7}\right) \\
& +\left(\left(\left(k U_{C}^{K}\right)^{*} P_{1}\right) \lambda_{7}\right)^{*}\left(\Upsilon_{C}^{K}\right)\left(\left(V_{C}^{K}\right) \lambda_{4}\right)+\left(\left(V_{C}^{K}\right) \lambda_{4}\right)^{T}\left(\Upsilon_{C}^{K}\right)^{T}\left(\left(\left(k U_{C}^{K}\right)^{*} P_{1}\right) \lambda_{7}\right) \\
& +\left(\left(-U_{D}^{T} P_{1}\right) \lambda_{5}\right)^{T}+\Upsilon_{D}\left(\left(V_{D}\right) \lambda_{7}\right)+\left(\left(V_{D}\right) \lambda_{7}\right)^{T} \Upsilon_{D}^{T}\left(\left(-U_{D}^{T} P_{1}\right) \lambda_{5}\right) \\
& +\left(\left(-U_{D}^{T} P_{1}\right) \lambda_{6}\right)^{T}+\Upsilon_{D}\left(\left(V_{D}\right) \lambda_{7}\right)+\left(\left(V_{D}\right) \lambda_{7}\right)^{T} \Upsilon_{D}^{T}\left(\left(-U_{D}^{T} P_{1}\right) \lambda_{6}\right) \\
& +\left(\left(-U_{D}^{T} P_{1} D_{0}\right) \lambda_{5}\right)^{T} \Upsilon_{D}\left(\left(V_{D}\right) \lambda_{6}\right)+\left(\left(V_{D}\right) \lambda_{6}\right)^{T} \Upsilon_{D}^{T}\left(\left(-U_{D}^{T} P_{1} D_{0}\right) \lambda_{5}\right) \\
& +\left(\left(-U_{D}^{T} P_{1} D_{0}\right) \lambda_{6}\right)^{T} \Upsilon_{D}\left(\left(V_{D}\right) \lambda_{5}\right)+\left(\left(V_{D}\right) \lambda_{5}\right)^{T} \Upsilon_{D}^{T}\left(\left(-U_{D}^{T} P_{1} D_{0}\right) \lambda_{6}\right) \\
& +\left(\left(-U_{D}^{T} P_{1} U_{D} \Upsilon_{D} V_{D}\right) \lambda_{5}\right)^{T} \Upsilon_{D}\left(\left(V_{D}\right) \lambda_{6}\right)+\left(\left(V_{D}\right) \lambda_{6}\right)^{T} \Upsilon_{D}^{T}\left(\left(-U_{D}^{T} P_{1} U_{D} \Upsilon_{D} V_{D}\right) \lambda_{5}\right) \text {, }
\end{aligned}
$$

where $\left(X \lambda_{i}\right)_{n^{2} \times 7 n}$ represents the block matrix, and the matrix $X_{n^{2} \times n}$ is in the $i$ th column, here $i=1,2, \ldots, 7, M_{11}=-P_{1} D_{0}-D_{0}^{T} P_{1}+\delta^{2} P_{3}+P_{2}+L\left(2 P_{2}+R\right) L$.

Using Lemma 2, we can only get $M<0$ if and only if there exist positive constants $\varepsilon_{i}$ $(i=1,2, \ldots, 34)$ such that

$$
\begin{aligned}
\bar{M}= & \left.\begin{array}{ccccccc}
M_{11} & \frac{3}{2} P_{1} A_{0} & \frac{3}{2} P_{1} B_{0} & \frac{3}{2} P_{1} C_{0} & D_{0}^{T} P_{1} D_{0} & -\frac{1}{2} P_{1} D_{0} & 0 \\
\star & P_{4}-2 P_{2} & 0 & 0 & 0 & 0 & \left(A_{0}\right)^{*} P_{1} \\
\star & \star & -P_{4} & 0 & 0 & 0 & \left(B_{0}\right)^{*} P_{1} \\
\star & \star & \star & -R & 0 & 0 & \left(C_{0}\right)^{*} P_{1} \\
\star & \star & \star & \star & -P_{3} & -D_{0}^{T} P_{1} D_{0} & -D_{0}^{T} P_{1} \\
\star & \star & \star & \star & \star & -P_{2} & -D_{0}^{T} P_{1} \\
\star & \star & \star & \star & \star & \star & -P_{1}-P_{1}
\end{array}\right) \\
& +\varepsilon_{1}^{-1}\left(\left(-U_{D}^{T} P_{1}\right) \lambda_{1}\right)^{T}\left(\left(-U_{D}^{T} P_{1}\right) \lambda_{1}\right)+\varepsilon_{1}\left(\left(V_{D}\right) \lambda_{1}\right)^{T}\left(\left(V_{D}\right) \lambda_{1}\right) \\
& +\varepsilon_{2}^{-1} \frac{9}{4}\left(\left(\left(U_{A}^{R}\right)^{T} P_{1}\right) \lambda_{1}\right)^{T}\left(\left(\left(U_{A}^{R}\right)^{T} P_{1}\right) \lambda_{1}\right)+\varepsilon_{2}\left(\left(V_{A}^{R}\right) \lambda_{2}\right)^{T}\left(\left(V_{A}^{R}\right) \lambda_{2}\right) \\
& +\varepsilon_{3}^{-1} \frac{9}{4}\left(\left(\left(i U_{A}^{I}\right)^{*} P_{1}\right) \lambda_{1}\right)^{*}\left(\left(\left(i U_{A}^{I}\right)^{*} P_{1}\right) \lambda_{1}\right)+\varepsilon_{3}\left(\left(V_{A}^{I}\right) \lambda_{2}\right)^{T}\left(\left(V_{A}^{I}\right) \lambda_{2}\right) \\
& +\varepsilon_{4}^{-1} \frac{9}{4}\left(\left(\left(j U_{A}^{J}\right)^{*} P_{1}\right) \lambda_{1}\right)^{*}\left(\left(\left(j U_{A}^{J}\right)^{*} P_{1}\right) \lambda_{1}\right)+\varepsilon_{4}\left(\left(V_{A}^{J}\right) \lambda_{2}\right)^{T}\left(\left(V_{A}^{J}\right) \lambda_{2}\right) \\
& +\varepsilon_{5}^{-1} \frac{9}{4}\left(\left(\left(k U_{A}^{K}\right)^{*} P_{1}\right) \lambda_{1}\right)^{*}\left(\left(\left(k U_{A}^{K}\right)^{*} P_{1}\right) \lambda_{1}\right)+\varepsilon_{5}\left(\left(V_{A}^{K}\right) \lambda_{2}\right)^{T}\left(\left(V_{A}^{K}\right) \lambda_{2}\right) \\
& +\varepsilon_{6}^{-1} \frac{9}{4}\left(\left(\left(U_{B}^{R}\right)^{T} P_{1}\right) \lambda_{1}\right)^{T}\left(\left(\left(U_{B}^{R}\right)^{T} P_{1}\right) \lambda_{1}\right)+\varepsilon_{6}\left(\left(V_{B}^{R}\right) \lambda_{3}\right)^{T}\left(\left(V_{B}^{R}\right) \lambda_{3}\right) \\
& +\varepsilon_{7}^{-1} \frac{9}{4}\left(\left(\left(i U_{B}^{I}\right)^{*} P_{1}\right) \lambda_{1}\right)^{*}\left(\left(\left(i U_{B}^{I}\right)^{*} P_{1}\right) \lambda_{1}\right)+\varepsilon_{7}\left(\left(V_{B}^{I}\right) \lambda_{3}\right)^{T}\left(\left(V_{B}^{I}\right) \lambda_{3}\right)
\end{aligned}
$$




$$
\begin{aligned}
& +\varepsilon_{8}^{-1} \frac{9}{4}\left(\left(\left(j U_{B}^{J}\right)^{*} P_{1}\right) \lambda_{1}\right)^{*}\left(\left(\left(j U_{B}^{J}\right)^{*} P_{1}\right) \lambda_{1}\right)+\varepsilon_{8}\left(\left(V_{B}^{J}\right) \lambda_{3}\right)^{T}\left(\left(V_{B}^{J}\right) \lambda_{3}\right) \\
& +\varepsilon_{9}^{-1} \frac{9}{4}\left(\left(\left(k U_{B}^{K}\right)^{*} P_{1}\right) \lambda_{1}\right)^{*}\left(\left(\left(k U_{B}^{K}\right)^{*} P_{1}\right) \lambda_{1}\right)+\varepsilon_{9}\left(\left(V_{B}^{K}\right) \lambda_{3}\right)^{T}\left(\left(V_{B}^{K}\right) \lambda_{3}\right) \\
& +\varepsilon_{10}^{-1} \frac{9}{4}\left(\left(\left(U_{C}^{R}\right)^{T} P_{1}\right) \lambda_{1}\right)^{T}\left(\left(\left(U_{C}^{R}\right)^{T} P_{1}\right) \lambda_{1}\right)+\varepsilon_{10}\left(\left(V_{C}^{R}\right) \lambda_{4}\right)^{T}\left(\left(V_{C}^{R}\right) \lambda_{4}\right) \\
& +\varepsilon_{11}^{-1} \frac{9}{4}\left(\left(\left(i U_{C}^{I}\right)^{*} P_{1}\right) \lambda_{1}\right)^{*}\left(\left(\left(i U_{C}^{I}\right)^{*} P_{1}\right) \lambda_{1}\right)+\varepsilon_{11}\left(\left(V_{C}^{I}\right) \lambda_{4}\right)^{T}\left(\left(V_{C}^{I}\right) \lambda_{4}\right) \\
& +\varepsilon_{12}^{-1} \frac{9}{4}\left(\left(\left(j U_{C}^{J}\right)^{*} P_{1}\right) \lambda_{1}\right)^{*}\left(\left(\left(j U_{C}^{J}\right)^{*} P_{1}\right) \lambda_{1}\right)+\varepsilon_{12}\left(\left(V_{C}^{J}\right) \lambda_{4}\right)^{T}\left(\left(V_{C}^{J}\right) \lambda_{4}\right) \\
& +\varepsilon_{13}^{-1} \frac{9}{4}\left(\left(\left(k U_{C}^{K}\right)^{*} P_{1}\right) \lambda_{1}\right)^{*}\left(\left(\left(k U_{C}^{K}\right)^{*} P_{1}\right) \lambda_{1}\right)+\varepsilon_{13}\left(\left(V_{C}^{K}\right) \lambda_{4}\right)^{T}\left(\left(V_{C}^{K}\right) \lambda_{4}\right) \\
& +\varepsilon_{14}^{-1}\left(\left(U_{D}^{T} P_{1} D_{0}\right) \lambda_{1}\right)^{T}\left(\left(U_{D}^{T} P_{1} D_{0}\right) \lambda_{1}\right)+\varepsilon_{14}\left(\left(V_{D}\right) \lambda_{5}\right)^{T}\left(\left(V_{D}\right) \lambda_{5}\right) \\
& +\varepsilon_{15}^{-1}\left(\left(U_{D}^{T} P_{1} D_{0}\right) \lambda_{5}\right)^{T}\left(\left(U_{D}^{T} P_{1} D_{0}\right) \lambda_{5}\right)+\varepsilon_{15}\left(\left(V_{D}\right) \lambda_{1}\right)^{T}\left(\left(V_{D}\right) \lambda_{1}\right) \\
& +\varepsilon_{16}^{-1}\left(\left(U_{D}^{T} P_{1} U_{D} \Upsilon_{D} V_{D}\right) \lambda_{1}\right)^{T}\left(\left(U_{D}^{T} P_{1} U_{D} \Sigma_{D} V_{D}\right) \lambda_{1}\right)+\varepsilon_{16}\left(\left(V_{D}\right) \lambda_{5}\right)^{T}\left(\left(V_{D}\right) \lambda_{5}\right) \\
& +\varepsilon_{17}^{-1} \frac{1}{4}\left(\left(-U_{D}^{T} P_{1}\right) \lambda_{1}\right)^{T}\left(\left(-U_{D}^{T} P_{1}\right) \lambda_{1}\right)+\varepsilon_{17}\left(\left(V_{D}\right) \lambda_{6}\right)^{T}\left(\left(V_{D}\right) \lambda_{6}\right) \\
& +\varepsilon_{18}^{-1}\left(\left(\left(U_{A}^{R}\right)^{T} P_{1}\right) \lambda_{7}\right)^{T}\left(\left(\left(U_{A}^{R}\right)^{T} P_{1}\right) \lambda_{7}\right)+\varepsilon_{18}\left(\left(V_{A}^{R}\right) \lambda_{2}\right)^{T}\left(\left(V_{A}^{R}\right) \lambda_{2}\right) \\
& +\varepsilon_{19}^{-1}\left(\left(\left(i U_{A}^{I}\right)^{*} P_{1}\right) \lambda_{7}\right)^{*}\left(\left(\left(i U_{A}^{I}\right)^{*} P_{1}\right) \lambda_{7}\right)+\varepsilon_{19}\left(\left(V_{A}^{I}\right) \lambda_{2}\right)^{T}\left(\left(V_{A}^{I}\right) \lambda_{2}\right) \\
& +\varepsilon_{20}^{-1}\left(\left(\left(j U_{A}^{J}\right)^{*} P_{1}\right) \lambda_{7}\right)^{*}\left(\left(\left(j U_{A}^{J}\right)^{*} P_{1}\right) \lambda_{7}\right)+\varepsilon_{20}\left(\left(V_{A}^{J}\right) \lambda_{2}\right)^{T}\left(\left(V_{A}^{J}\right) \lambda_{2}\right) \\
& +\varepsilon_{21}^{-1}\left(\left(\left(k U_{A}^{K}\right)^{*} P_{1}\right) \lambda_{7}\right)^{*}\left(\left(\left(k U_{A}^{K}\right)^{*} P_{1}\right) \lambda_{7}\right)+\varepsilon_{21}\left(\left(V_{A}^{K}\right) \lambda_{2}\right)^{T}\left(\left(V_{A}^{K}\right) \lambda_{2}\right) \\
& +\varepsilon_{22}^{-1}\left(\left(\left(U_{B}^{R}\right)^{T} P_{1}\right) \lambda_{7}\right)^{T}\left(\left(\left(U_{B}^{R}\right)^{T} P_{1}\right) \lambda_{7}\right)+\varepsilon_{22}\left(\left(V_{B}^{R}\right) \lambda_{3}\right)^{T}\left(\left(V_{B}^{R}\right) \lambda_{3}\right) \\
& +\varepsilon_{23}^{-1}\left(\left(\left(i U_{B}^{I}\right)^{*} P_{1}\right) \lambda_{7}\right)^{*}\left(\left(\left(i U_{B}^{I}\right)^{*} P_{1}\right) \lambda_{7}\right)+\varepsilon_{23}\left(\left(V_{B}^{I}\right) \lambda_{3}\right)^{T}\left(\left(V_{B}^{I}\right) \lambda_{3}\right) \\
& +\varepsilon_{24}^{-1}\left(\left(\left(j U_{B}^{J}\right)^{*} P_{1}\right) \lambda_{7}\right)^{*}\left(\left(\left(j U_{B}^{J}\right)^{*} P_{1}\right) \lambda_{7}\right)+\varepsilon_{24}\left(\left(V_{B}^{J}\right) \lambda_{3}\right)^{T}\left(\left(V_{B}^{J}\right) \lambda_{3}\right) \\
& +\varepsilon_{25}^{-1}\left(\left(\left(k U_{B}^{K}\right)^{*} P_{1}\right) \lambda_{7}\right)^{*}\left(\left(\left(k U_{B}^{K}\right)^{*} P_{1}\right) \lambda_{7}\right)+\varepsilon_{25}\left(\left(V_{B}^{K}\right) \lambda_{3}\right)^{T}\left(\left(V_{B}^{K}\right) \lambda_{3}\right) \\
& +\varepsilon_{26}^{-1}\left(\left(\left(U_{C}^{R}\right)^{T} P_{1}\right) \lambda_{7}\right)^{T}\left(\left(\left(U_{C}^{R}\right)^{T} P_{1}\right) \lambda_{7}\right)+\varepsilon_{26}\left(\left(V_{C}^{R}\right) \lambda_{4}\right)^{T}\left(\left(V_{C}^{R}\right) \lambda_{4}\right) \\
& +\varepsilon_{27}^{-1}\left(\left(\left(i U_{C}^{I}\right)^{*} P_{1}\right) \lambda_{7}\right)^{*}\left(\left(\left(i U_{C}^{I}\right)^{*} P_{1}\right) \lambda_{7}\right)+\varepsilon_{27}\left(\left(V_{C}^{I}\right) \lambda_{4}\right)^{T}\left(\left(V_{C}^{I}\right) \lambda_{4}\right) \\
& +\varepsilon_{28}^{-1}\left(\left(\left(j U_{C}^{J}\right)^{*} P_{1}\right) \lambda_{7}\right)^{*}\left(\left(\left(j U_{C}^{J}\right)^{*} P_{1}\right) \lambda_{7}\right)+\varepsilon_{28}\left(\left(V_{C}^{J}\right) \lambda_{4}\right)^{T}\left(\left(V_{C}^{J}\right) \lambda_{4}\right) \\
& +\varepsilon_{29}^{-1}\left(\left(\left(k U_{C}^{K}\right)^{*} P_{1}\right) \lambda_{7}\right)^{*}\left(\left(\left(k U_{C}^{K}\right)^{*} P_{1}\right) \lambda_{7}\right)+\varepsilon_{29}\left(\left(V_{C}^{K}\right) \lambda_{4}\right)^{T}\left(\left(V_{C}^{K}\right) \lambda_{4}\right) \\
& +\varepsilon_{30}^{-1}\left(\left(-U_{D}^{T} P_{1}\right) \lambda_{5}\right)^{T}\left(\left(-U_{D}^{T} P_{1}\right) \lambda_{5}\right)+\varepsilon_{30}\left(\left(V_{D}\right) \lambda_{7}\right)^{T}\left(\left(V_{D}\right) \lambda_{7}\right) \\
& +\varepsilon_{31}^{-1}\left(\left(-U_{D}^{T} P_{1}\right) \lambda_{6}\right)^{T}\left(\left(-U_{D}^{T} P_{1}\right) \lambda_{6}\right)+\varepsilon_{31}\left(\left(V_{D}\right) \lambda_{7}\right)^{T}\left(\left(V_{D}\right) \lambda_{7}\right) \\
& +\varepsilon_{32}^{-1}\left(\left(-U_{D}^{T} P_{1} D_{0}\right) \lambda_{5}\right)^{T}\left(\left(-U_{D}^{T} P_{1} D_{0}\right) \lambda_{5}\right)+\varepsilon_{32}\left(\left(V_{D}\right) \lambda_{6}\right)^{T}\left(\left(V_{D}\right) \lambda_{6}\right) \\
& +\varepsilon_{33}^{-1}\left(\left(-U_{D}^{T} P_{1} D_{0}\right) \lambda_{6}\right)^{T}\left(\left(-U_{D}^{T} P_{1} D_{0}\right) \lambda_{6}\right)+\varepsilon_{33}\left(\left(V_{D}\right) \lambda_{5}\right)^{T}\left(\left(V_{D}\right) \lambda_{5}\right) \\
& +\varepsilon_{34}^{-1}\left(\left(-U_{D}^{T} P_{1} U_{D} \Sigma_{D} V_{D}\right) \lambda_{5}\right)^{T}\left(\left(-U_{D}^{T} P_{1} U_{D} \Sigma_{D} V_{D}\right) \lambda_{5}\right)+\varepsilon_{34}\left(\left(V_{D}\right) \lambda_{6}\right)^{T}\left(\left(V_{D}\right) \lambda_{6}\right) \\
& <0 \text {. }
\end{aligned}
$$

Applying Lemma 3, we obtain that (9) is equivalent to (3), and we know $\bar{M}=S_{11}$ $S_{12} S_{22}^{-1} S_{21}$, also $\Xi=\left(\begin{array}{ll}S_{11} & S_{12} \\ S_{21} & S_{22}\end{array}\right)$. It is clear that $S_{22}<0$, where $\left(S_{11}\right)_{7 n \times 7 n},\left(S_{12}\right)_{7 n \times 34 n},\left(S_{21}\right)_{34 n \times 7 n}$, 
$\left(S_{22}\right)_{34 n \times 34 n}$ are the partitioned matrices of the matrix $\Xi$. Therefore, the proof of this theorem is turned into the proof that model (1) has the global robust stability of the unique equilibrium point if $\bar{M}<0$.

Step 2: Under the condition $M<0$, we prove that model (1) has a unique equilibrium point. Let $\check{q}$ be an equilibrium point of the system (1), and $\check{q}$ satisfies

$$
-D \check{q}+A g(\check{q})+B g(\check{q})+C g(\check{q})+J=0 .
$$

Let $G(q)=-D q+A g(q)+B g(q)+C g(q)+J$.

In the following, we prove that map $G$ is a homeomorphism, where $G: \mathbb{H}^{n} \rightarrow \mathbb{H}^{n}$.

On the one hand, we prove that $G(q)$ is an injective map on $\mathbb{H}^{n}$.

Assuming there are $q_{1}, q_{2} \in \mathbb{H}^{n}$ with $q_{1} \neq q_{2}$ such that $G\left(q_{1}\right)=G\left(q_{2}\right)$, we can get

$$
-D\left(q_{1}-q_{2}\right)+A\left(g\left(q_{1}\right)-g\left(q_{2}\right)\right)+B\left(g\left(q_{1}\right)-g\left(q_{2}\right)\right)+C\left(g\left(q_{1}\right)-g\left(q_{2}\right)\right)=0 .
$$

Left-multiplying both sides of (11) by $\frac{9}{4}\left(q_{1}-q_{2}\right)^{*} P_{1}$, we get

$$
\begin{aligned}
0= & -\left(q_{1}-q_{2}\right)^{*} \frac{9}{4} P_{1} D\left(q_{1}-q_{2}\right)+\left(q_{1}-q_{2}\right)^{*} \frac{9}{4} P_{1} A\left(g\left(q_{1}\right)-g\left(q_{2}\right)\right) \\
& +\left(q_{1}-q_{2}\right)^{*} \frac{9}{4} P_{1} B\left(g\left(q_{1}\right)-g\left(q_{2}\right)\right)+\left(q_{1}-q_{2}\right)^{*} \frac{9}{4} P_{1} C\left(g\left(q_{1}\right)-g\left(q_{2}\right)\right) .
\end{aligned}
$$

By taking the conjugate transpose on both sides of (12) leads to

$$
\begin{aligned}
0= & -\left(q_{1}-q_{2}\right)^{*} \frac{9}{4} D^{*} P_{1}\left(q_{1}-q_{2}\right)+\left(g\left(q_{1}\right)-g\left(q_{2}\right)\right)^{*} \frac{9}{4} A^{*} P_{1}\left(q_{1}-q_{2}\right) \\
& +\left(g\left(q_{1}\right)-g\left(q_{2}\right)\right)^{*} \frac{9}{4} B^{*} P_{1}\left(q_{1}-q_{2}\right) \\
& +\left(g\left(q_{1}\right)-g\left(q_{2}\right)\right)^{*} \frac{9}{4} C^{*} P_{1}\left(q_{1}-q_{2}\right) .
\end{aligned}
$$

Adding (12) and (13) brings about

$$
\begin{aligned}
0= & -\left(q_{1}-q_{2}\right)^{*}\left(-\frac{9}{4} P_{1} D-\frac{9}{4} D^{*} P_{1}\right)\left(q_{1}-q_{2}\right) \\
& +\left(q_{1}-q_{2}\right)^{*} \frac{9}{4} P_{1} A+\left(g\left(q_{1}\right)-g\left(q_{2}\right)\right)+\left(g\left(q_{1}\right)-g\left(q_{2}\right)\right)^{*} \frac{9}{4} A^{*} P_{1}\left(q_{1}-q_{2}\right) \\
& +\left(q_{1}-q_{2}\right)^{*} \frac{9}{4} P_{1} B+\left(g\left(q_{1}\right)-g\left(q_{2}\right)\right)+\left(g\left(q_{1}\right)-g\left(q_{2}\right)\right)^{*} \frac{9}{4} B^{*} P_{1}\left(q_{1}-q_{2}\right) \\
& +\left(q_{1}-q_{2}\right)^{*} \frac{9}{4} P_{1} C+\left(g\left(q_{1}\right)-g\left(q_{2}\right)\right) \\
& +\left(g\left(q_{1}\right)-g\left(q_{2}\right)\right)^{*} \frac{9}{4} C^{*} P_{1}\left(q_{1}-q_{2}\right) .
\end{aligned}
$$

From $M<0$, we know

$$
P_{4}-P_{2}<0, \quad-P_{4}<0, \quad-\left(P_{2}+\delta^{2} P_{3}\right)<0,
$$




$$
\left(\begin{array}{cccc}
-P_{1} D-D^{T} P_{1}+P_{2}+\delta^{2} P_{3}+L\left(R+2 P_{2}\right) L & \frac{3}{2} P_{1} A & \frac{3}{2} P_{1} B & \frac{3}{2} P_{1} C \\
\star & P_{4}-2 P_{2} & 0 & 0 \\
\star & \star & -P_{4} & 0 \\
\star & \star & \star & -R
\end{array}\right)<0
$$

It follows from (15) and (16) that

$$
\left(\begin{array}{cccc}
-P_{1} D-D^{T} P_{1}+L\left(R+2 P_{2}\right) L & \frac{3}{2} P_{1} A & \frac{3}{2} P_{1} B & \frac{3}{2} P_{1} C \\
\star & P_{4}-2 P_{2} & 0 & 0 \\
\star & \star & -P_{4} & 0 \\
\star & \star & \star & -R
\end{array}\right)<0
$$

By Lemma 3, we have

$$
\begin{aligned}
& -P_{1} D-D^{T} P_{1}+L\left(2 P_{2}+R\right) L+P_{1} A \frac{9}{4}\left(2 P_{2}-P_{4}\right)^{-1} A^{*} P_{1} \\
& +P_{1} B \frac{9}{4} P_{4}^{-1} B^{*} P_{1}+P_{1} C \frac{9}{4} R^{-1} C^{*} P_{1}<0 .
\end{aligned}
$$

Using Lemma 4 and Assumption $\left(\mathbf{A}_{1}\right)$, for the positive definiteness of $2 P_{2}-P_{4}, P_{4}$ and $R$, we have from (14) that

$$
\begin{aligned}
0 \leq & \left(q_{1}-q_{2}\right)^{*}\left[-\frac{9}{4} P_{1} D-\frac{9}{4} D^{*} P_{1}+P_{1} A \frac{81}{16}\left(2 P_{2}-P_{4}\right)^{-1} A^{*} P_{1}\right. \\
& \left.+P_{1} B \frac{81}{16} P_{4}^{-1} B^{*} P_{1}+P_{1} C \frac{81}{16} R^{-1} C^{*} P_{1}\right]\left(q_{1}-q_{2}\right) \\
& +\left(g\left(q_{1}\right)-g\left(q_{2}\right)\right)^{*}\left(2 P_{2}+R\right)\left(g\left(q_{1}\right)-g\left(q_{2}\right)\right) \\
\leq & \left(q_{1}-q_{2}\right)^{*}\left[-\frac{9}{4} P_{1} D-\frac{9}{4} D^{*} P_{1}+P_{1} A \frac{81}{16}\left(2 P_{2}-P_{4}\right)^{-1} A^{*} P_{1}\right. \\
& \left.+P_{1} B \frac{81}{16} P_{4}^{-1} B^{*} P_{1}+P_{1} C \frac{81}{16} R^{-1} C^{*} P_{1}+L\left(2 P_{2}+R\right) L\right]\left(q_{1}-q_{2}\right) \\
= & \left(q_{1}-q_{2}\right)^{*}\left[-P_{1} D-D^{*} P_{1}+P_{1} A \frac{9}{4}\left(2 P_{2}-P_{4}\right)^{-1} A^{*} P_{1}\right. \\
& \left.+P_{1} B \frac{9}{4} P_{4}^{-1} B^{*} P_{1}+P_{1} C \frac{9}{4} R^{-1} C^{*} P_{1}+\frac{4}{9} L\left(2 P_{2}+R\right) L\right]\left(q_{1}-q_{2}\right) .
\end{aligned}
$$

Since $L\left(R+2 P_{2}\right) L>0$ and from (18), we have

$$
\begin{aligned}
& -P_{1} D-D^{*} P_{1}+\frac{4}{9} L\left(2 P_{2}+R\right) L+P_{1} A \frac{9}{4}\left(2 P_{2}-P_{4}\right)^{-1} A^{*} P_{1} \\
& +P_{1} B \frac{9}{4} P_{4}^{-1} B^{*} P_{1}+P_{1} C \frac{9}{4} R^{-1} C^{*} P_{1}<0
\end{aligned}
$$

From (19) and (20), we get $q_{1}=q_{2}$, which contradicts our assumption. Therefore, $G(q)$ is an injective map on $\mathbb{H}^{n}$.

On the other hand, we prove that $\|G(q)\| \rightarrow+\infty$ as $\|q\| \rightarrow+\infty$. Indeed,

$$
G(q)-G(0)=-D q+A(g(q)-g(0))+B(g(q)-g(0))+C(g(q)-g(0)) .
$$


Left-multiplying by $\frac{9}{4} q^{*} P_{1}$ both sides of (21) leads to

$$
\begin{aligned}
\frac{9}{4} q^{*} P_{1}(G(q)-G(0))= & -\frac{9}{4} q^{*} P_{1} D q+\frac{9}{4} q^{*} P_{1} A(g(q)-g(0)) \\
& +\frac{9}{4} q^{*} P_{2} B(g(q)-g(0))+\frac{9}{4} q^{*} P_{2} C(g(q)-g(0))
\end{aligned}
$$

Taking the conjugate transpose of equality (22), we obtain that

$$
\begin{aligned}
\frac{9}{4}(G(q)-G(0))^{*} P_{1} q= & -\frac{9}{4} q^{*} D^{*} P_{1} q+(g(q)-g(0))^{*} A^{*} P_{1} \\
& +\frac{9}{4}(g(q)-g(0))^{*} B^{*} P_{1} q+\frac{9}{4}(g(q)-g(0))^{*} C^{*} P_{1} q .
\end{aligned}
$$

Summing (22) and (23) leads to

$$
\begin{aligned}
q^{*} P_{1}(G(q)-G(0))+(G(q)-G(0))^{*} P_{1} q \\
=q^{*}\left(-\frac{9}{4} P_{1} D-\frac{9}{4} D^{*} P_{1}\right) q \\
\quad+q^{*} \frac{9}{4} P_{1} A(g(q)-g(0))+(g(q)-g(0))^{*} \frac{9}{4} A^{*} P_{1} q \\
\quad+q^{*} \frac{9}{4} P_{1} B(g(q)-g(0))+(g(q)-g(0))^{*} \frac{9}{4} B^{*} P_{1} q \\
\quad+q^{*} \frac{9}{4} P_{1} C(g(q)-g(0))+(g(q)-g(0))^{*} \frac{9}{4} C^{*} P_{1} q .
\end{aligned}
$$

Similar to proving the injectivity of the map, we can get

$$
\begin{aligned}
q^{*} P_{1}(G(q)-G(0))+(G(q)-G(0))^{*} P_{1} q \\
\leq q^{*}\left[-P_{1} D-D^{*} P_{1}+\frac{9}{4} P_{1} A\left(2 P_{2}-P_{4}\right)^{-1} A^{*} P_{1}\right. \\
\left.\quad+\frac{9}{4} P_{1} B P_{4}^{-1} B^{*} P_{1}+4 P_{1} C R^{-1} C^{*} P_{1}+\frac{4}{9} L\left(R+2 P_{2}\right) L\right] q \\
\leq-\lambda_{\min }(-\Theta)\|q\|^{2},
\end{aligned}
$$

where

$$
\begin{aligned}
\Theta= & -P_{1} D-D^{*} P_{1}+\frac{9}{4} P_{1} A\left(2 P_{2}-P_{4}\right)^{-1} A^{*} P_{1}+\frac{9}{4} P_{1} B P_{4}^{-1} B^{*} P_{1} \\
& +\frac{9}{4} P_{1} C R^{-1} C^{*} P_{1}+\frac{4}{9} L\left(R+2 P_{2}\right) L<0 .
\end{aligned}
$$

According to Cauchy-Schwarz inequality,

$$
\begin{aligned}
\lambda_{\min }(-\Theta)\|q\|^{2} & \leq-q^{*} P_{1}(G(q)-G(0))+(G(q)-G(0))^{*} P_{1} q \\
& =-2 \operatorname{Re}\left(q^{*} P_{1}(G(q)-G(0))\right) \\
& \leq 2\left|q^{*} P_{1}(G(q)-G(0))\right|
\end{aligned}
$$




$$
\begin{aligned}
& \leq 2\|q\| \cdot\left\|P_{1}\right\| \cdot\|G(q)-G(0)\| \\
& \leq 2\|q\| \cdot\left\|P_{2}\right\| \cdot(\|G(q)\|+\|G(0)\|) .
\end{aligned}
$$

Therefore, $\|G(q)\| \rightarrow+\infty$ as $\|q\| \rightarrow+\infty$, so we know that $G(q)$ is a homeomorphic map on $\mathbb{H}^{n}$ by Lemma 5 . Thus, system (1) has a unique equilibrium point.

Step 3: We prove that the equilibrium point enjoys global asymptotical robust stability. From the previous proof, we know that system (1) has a unique equilibrium point $\check{q}$. For convenience letting $q(\tilde{q})=q(t)-\check{q}$, system (1) can be rewritten as

$$
\left\{\begin{aligned}
\dot{\tilde{q}}(t)= & -D \tilde{q}(t-\delta)+A f(\tilde{q}(t))+B f(\tilde{q}(t-\tau)) \\
& +C \int_{-\infty}^{t} K(t-s) f(\tilde{q}(s)) d s, \quad t>0, t \neq t_{k} . \\
\Delta \tilde{q}= & M_{k}\left(\tilde{q}\left(t_{k}^{-}\right)\right), \quad k=1,2, \ldots,
\end{aligned}\right.
$$

where $f(\tilde{q}(t))=g(q(t))-g(\check{q}), f(\tilde{q}(t-\tau))=g(q(t-\tau))-g(\check{q})$. In the meantime, the initial condition (2) can be transformed into

$$
\tilde{q}(s)=\tilde{\vartheta}(s), \quad s \in[-\rho, 0],
$$

where $\tilde{\vartheta}(s)=\vartheta(s)-\check{q} \in C\left([-\rho, 0], \mathbb{H}^{n}\right)$.

Consider the following Lyapunov function:

$$
V(\tilde{q}(t))=V_{1}(\tilde{q}(t))+V_{2}(\tilde{q}(t))+V_{3}(\tilde{q}(t))+V_{4}(\tilde{q}(t))+V_{5}(\tilde{q}(t))+V_{6}(\tilde{q}(t)),
$$

where

$$
\begin{aligned}
& V_{1}(\tilde{q}(t))=\left(\tilde{q}(t)-D \int_{t-\delta}^{t} \tilde{q}(s) d s\right)^{*} P_{1}\left(\tilde{q}(t)-D \int_{t-\delta}^{t} \tilde{q}(s) d s\right), \\
& V_{2}(\tilde{q}(t))=\int_{t-\delta}^{t} \tilde{q}^{*}(s) P_{2} \tilde{q}(s) d s, \\
& V_{3}(\tilde{q}(t))=\delta \int_{0}^{\delta} \int_{t-u}^{t} \tilde{q}^{*}(s) P_{3} \tilde{q}(s) d s d u, \\
& V_{4}(\tilde{q}(t))=\int_{t-\tau}^{t} f^{*}(\tilde{q}(s)) P_{4} f(\tilde{q}(s)) d s, \\
& V_{5}(\tilde{q}(t))=\sum_{j=1}^{n} r_{j} \int_{0}^{\infty} K(s) \int_{t-s}^{t} f_{j}^{*}\left(\tilde{q}_{j}(t)\right) f_{j}\left(\tilde{q}_{j}(t)\right) d t d s, \\
& V_{6}(\tilde{q}(t))=\frac{1}{2} \tilde{q}^{*}(t) P_{1} \tilde{q}(t),
\end{aligned}
$$

where $r_{j}$ is the principal diagonal element of $R$ and $R=\operatorname{diag}\left(r_{1}, r_{2}, \ldots, r_{n}\right)$.

When $t \neq t_{k}$, taking the time derivative of $V_{1}(\tilde{q}(t)), V_{2}(\tilde{q}(t)), V_{3}(\tilde{q}(t)), V_{4}(\tilde{q}(t)), V_{5}(\tilde{q}(t))$, and $V_{6}(\tilde{q}(t))$, we can get

$$
\begin{aligned}
\dot{V}_{1}(\tilde{q}(t))= & \left.(\tilde{q}(t))-D \int_{t-\delta}^{t} \tilde{q}(s) d s\right)^{*} P_{1}(\dot{\tilde{q}}(t)-D \tilde{q}(t)+D \tilde{q}(t-\delta)) \\
& +\left(\dot{\tilde{q}}(t)-D \tilde{q}(t)+D \tilde{q}(t-\delta)^{*} P_{1}(\tilde{q}(t))-D \int_{t-\delta}^{t} \tilde{q}(s) d s\right)
\end{aligned}
$$




$$
\begin{aligned}
& \left.=(\tilde{q}(t))-D \int_{t-\delta}^{t} \tilde{q}(s) d s\right)^{*} P_{1}(A f(\tilde{q}(t)) \\
& +B f(\tilde{q}(t-\tau))+C \int_{-\infty}^{t} K(t-s) f(\tilde{q}(s)) d s \\
& -D \tilde{q}(t))+\left(A f(\tilde{q}(t))+B f(\tilde{q}(t-\tau))+C \int_{-\infty}^{t} K(t-s) f(\tilde{q}(s)) d s\right. \\
& -D \tilde{q}(t))^{*} P_{1}\left(\tilde{q}(t)-D \int_{t-\delta}^{t} \tilde{q}(s) d s\right) \\
& =\tilde{q}^{*}(t) P_{1} A f(\tilde{q}(t))+\tilde{q}^{*}(t) P_{1} B f(\tilde{q}(t-\tau)) \\
& +\tilde{q}^{*}(t) P_{1} C \int_{-\infty}^{t} K(t-s) f(\tilde{q}(s)) d s-\tilde{q}^{*}(t) P_{1} D \tilde{q}(t) \\
& +f^{*}(\tilde{q}(t)) A^{*} P_{1} \tilde{q}(t)+f^{*}(\tilde{q}(t-\tau)) B^{*} P_{1} \tilde{q}(t) \\
& +\left(\int_{-\infty}^{t} K(t-s) f(\tilde{q}(s)) d s\right)^{*} C^{*} P_{1} \tilde{q}(t)-\tilde{q}^{*}(t) D^{T} P_{1} \tilde{q}(t) \\
& -\left(\int_{t-\delta}^{t} \tilde{q}(s) d s\right)^{*} D^{T} P_{1} A f(\tilde{q}(t)) \\
& -\left(\int_{t-\delta}^{t} \tilde{q}(s) d s\right)^{*} D^{T} P_{1} B f(\tilde{q}(t-\tau)) \\
& -\left(\int_{t-\delta}^{t} \tilde{q}(s) d s\right)^{*} D^{T} P_{1} C \int_{t}^{+\infty} K(t-s) f(\tilde{q}(s)) d s \\
& +\left(\int_{t-\delta}^{t} \tilde{q}(s) d s\right)^{*} D^{T} P_{1} D \tilde{q}(t)-f^{*}(\tilde{q}(t)) A^{*} P_{1} D \int_{t-\delta}^{t} \tilde{q}(s) d s \\
& -f^{*}(\tilde{q}(t-\tau)) B^{*} P_{1} D \int_{t-\delta}^{t} \tilde{q}(s) d s \\
& -\left(\int_{-\infty}^{t} K(t-s) f(\tilde{q}(s)) d s\right)^{*} C^{*} P_{1} D \int_{t-\delta}^{t} \tilde{q}(s) d s \\
& +\tilde{q}^{*}(t) D^{T} P_{1} D \int_{t-\delta}^{t} \tilde{q}(s) d s, \\
& \dot{V}_{2}(\tilde{q}(t))=\tilde{q}(t)^{*}(t) P_{2} \tilde{q}(t)-\tilde{q}^{*}(t-\delta) P_{2} \tilde{q}(t-\delta), \\
& \dot{V}_{3}(\tilde{q}(t))=\delta^{2} \tilde{q}(t)^{*}(t) P_{3} \tilde{q}(t)-\delta \int_{0}^{\delta} \tilde{q}^{*}(t-u) P_{3} \tilde{q}(t-u) d u \\
& =\delta^{2} \tilde{q}(t)^{*}(t) P_{3} \tilde{q}(t)-\delta \int_{t-\delta}^{t} \tilde{q}^{*}(s) P_{3} \tilde{q}(s) d s .
\end{aligned}
$$

Using Lemma 5, we have

$$
\begin{aligned}
\dot{V}_{3}(\tilde{q}(t)) \leq & \delta^{2} \tilde{q}(t)^{*}(t) P_{3} \tilde{q}(t)-\left(\int_{t-\delta}^{t} \tilde{q}^{*}(s) d s\right)^{*} P_{3}\left(\int_{t-\delta}^{t} \tilde{q}^{*}(s) d s\right), \\
\dot{V}_{4}(\tilde{q}(t))= & f^{*}(\tilde{q}(t)) P_{4} f(\tilde{q}(t))-f^{*}(\tilde{q}(t-\tau)) P_{4} f(\tilde{q}(t-\tau)), \\
\dot{V}_{5}(\tilde{q}(t))= & \sum_{j=1}^{n} r_{j} \int_{0}^{\infty} K(s) f_{j}^{*}\left(\tilde{q}_{j}(t)\right) f_{j}\left(\tilde{q}_{j}(t)\right) d s \\
& -\sum_{j=1}^{n} r_{j} \int_{0}^{\infty} K(s) f_{j}^{*}\left(\tilde{q}_{j}(t-s)\right) f_{j}\left(\tilde{q}_{j}(t-s)\right) d s .
\end{aligned}
$$


Magnifying the equation for $\dot{V}_{5}(\tilde{q}(t))$ by using assumption $\left(\mathbf{A}_{1}\right)$ yields

$$
\begin{aligned}
\dot{V}_{5}(\tilde{q}(t)) \leq & \tilde{q}^{*}(t) L R L \tilde{q}(t) \\
& -\sum_{j=1}^{n} r_{j} \int_{0}^{\infty} K(s) f_{j}^{*}\left(\tilde{q}_{j}(t-s)\right) d s \int_{0}^{\infty} K(s) f_{j}\left(\tilde{q}_{j}(t-s)\right) d s \\
\leq & \tilde{q}^{*}(t) L R L \tilde{q}(t) \\
& -\left(\int_{-\infty}^{t} K(s) f_{j}\left(\tilde{q}_{j}(t-s)\right) d s\right)^{*} R\left(\int_{-\infty}^{t} K(s) f_{j}\left(\tilde{q}_{j}(t-s)\right) d s\right), \\
\dot{V}_{6}(\tilde{q}(t))= & \frac{1}{2}\left[\dot{\tilde{q}}^{*}(t) P_{1} \tilde{q}(t)+\tilde{q}^{*}(t) P_{1} \dot{\tilde{q}}(t)\right] \\
= & \frac{1}{2}[(-D \tilde{q}(t-\delta)+A f(\check{q}(t))+B f(\tilde{q}(t-\tau)) \\
& \left.+C \int_{-\infty}^{t} K(t-s) f(\tilde{q}(s)) d s\right)^{*} P_{1} \tilde{q}(t)+\tilde{q}^{*}(t) P_{1}(-D \tilde{q}(t-\delta) \\
& \left.\left.+A f(\check{q}(t))+B f(\tilde{q}(t-\tau))+C \int_{-\infty}^{t} K(t-s) f(\tilde{q}(s)) d s\right)\right] \\
= & \frac{1}{2}\left[-\tilde{q}^{*}(t-\delta) D^{T} P_{1} \tilde{q}(t)+f^{*}(\check{q}(t)) A^{*} P_{1} \tilde{q}(t)\right. \\
& +f^{*}(\check{q}(t-\tau)) B^{*} P_{1} \tilde{q}(t)+\left(\int_{-\infty}^{t} K(t-s) f(\tilde{q}(s)) d s\right) C^{*} P_{1} \tilde{q}(t) \\
& -\tilde{q}^{*}(t) P_{1} D \tilde{q}(t-\delta)+\tilde{q}^{*}(t) P_{1} A f(\tilde{q}(t)) \\
& \left.+\tilde{q}^{*}(t) P_{1} B f(\tilde{q}(t-\tau))+\tilde{q}^{*}(t) P_{1} C \int_{-\infty}^{t} K(t-s) f(\tilde{q}(s)) d s\right] .
\end{aligned}
$$

In addition, for the real-valued diagonal matrix $2 P_{2}$, using assumption $\left(\mathbf{A}_{1}\right)$, we can get

$$
0 \leq \tilde{q}^{*}(t) L 2 P_{2} L \tilde{q}(t)-f^{*}(\tilde{q}(t)) 2 P_{2} f(\tilde{q}(t)) .
$$

From equation (25), we have

$$
\begin{aligned}
0= & \left(P_{1} \dot{\tilde{q}}(t)+P_{1} D \int_{t-\delta}^{t} \tilde{q}(s) d s\right)^{*}(-\dot{\tilde{q}}(t)-D \tilde{q}(t-\delta)+A f(\check{q}(t)) \\
& \left.+B f(\tilde{q}(t-\tau))+C \int_{-\infty}^{t} K(t-s) f(\tilde{q}(s)) d s\right) \\
& +(-\dot{\tilde{q}}(t)-D \tilde{q}(t-\delta)+A f(\check{q}(t))+B f(\tilde{q}(t-\tau)) \\
& \left.+C \int_{-\infty}^{t} K(t-s) f(\tilde{q}(s)) d s\right)^{*}\left(P_{1} \dot{\tilde{q}}(t)+P_{1} D \int_{t-\delta}^{t} \tilde{q}(s) d s\right) \\
= & \dot{\tilde{q}}^{*}(t) P_{1} \dot{\tilde{q}}(t)-\dot{\tilde{q}}^{*}(t) P_{1} D \tilde{q}(t-\delta)+\dot{\tilde{q}}^{*}(t) P_{1} A f(\check{q}(t))+\dot{\tilde{q}}^{*}(t) P_{1} B f(\tilde{q}(t-\tau)) \\
& +\dot{\tilde{q}}^{*}(t) P_{1} C \int_{-\infty}^{t} K(t-s) f(\tilde{q}(s)) d s-\left(\int_{t-\delta}^{t} \tilde{q}(s) d s\right)^{*} D^{T} P_{1} \dot{\tilde{q}}(t) \\
& -\left(\int_{t-\delta}^{t} \tilde{q}(s) d s\right)^{*} D^{T} P_{1} D \tilde{q}(t-\delta)+\left(\int_{t-\delta}^{t} \tilde{q}(s) d s\right)^{*} D^{T} P_{1} A f(\tilde{q}(t))
\end{aligned}
$$




$$
\begin{aligned}
& +\left(\int_{t-\delta}^{t} \tilde{q}(s) d s\right)^{*} D^{T} P_{1} B f(\tilde{q}(t-\tau)) \\
& +\left(\int_{t-\delta}^{t} \tilde{q}(s) d s\right)^{*} D^{T} P_{1} C \int_{-\infty}^{t} K(t-s) f(\tilde{q}(s)) d s \\
& -\dot{\tilde{q}}^{*}(t) P_{1} \dot{\tilde{q}}(t)-\dot{\tilde{q}}(t-\delta) D^{T} P_{1} \dot{\tilde{q}}(t) \\
& +f^{*}(\check{q}(t)) A^{*} P_{1} \dot{\tilde{q}}(t)+f^{*}(\tilde{q}(t-\tau)) B^{*} P_{1} \dot{\tilde{q}}(t) \\
& +\left(\int_{-\infty}^{t} K(t-s) f(\tilde{q}(s)) d s\right)^{*} C^{*} P_{1} \dot{\tilde{q}}(t) \\
& -\dot{\tilde{q}}^{*}(t) P_{1} D \int_{t-\delta}^{t} \tilde{q}(s) d s-\tilde{q}^{*}(t-\delta) D^{T} P_{1} D \int_{t-\delta}^{t} \tilde{q}(s) d s \\
& +f^{*}(\check{q}(t)) A^{*} P_{1} \int_{t-\delta}^{t} \tilde{q}(s) d s+f^{*}(\tilde{q}(t-\tau)) B^{*} P_{1} \int_{t-\delta}^{t} \tilde{q}(s) d s \\
& +\left(\int_{-\infty}^{t} K(t-s) f(\tilde{q}(s)) d s\right)^{*} C^{*} P_{1} D \int_{t-\delta}^{t} \tilde{q}(s) d s .
\end{aligned}
$$

So from (33)-(39) it follows that

$$
\begin{aligned}
\dot{V}(\tilde{q}(t)) \leq & \tilde{q}^{*}(t) \frac{3}{2} P_{1} A f(\tilde{q}(t))+\tilde{q}^{*}(t) \frac{3}{2} P_{1} B f(\tilde{q}(t-\tau)) \\
& +\tilde{q}^{*}(t) \frac{3}{2} P_{1} C \int_{-\infty}^{t} K(t-s) f(\tilde{q}(s)) d s-\tilde{q}^{*}(t) P_{1} D \tilde{q}(t) \\
& +f^{*}(\tilde{q}(t)) \frac{3}{2} A^{*} P_{1} \tilde{q}(t)+f^{*}(\tilde{q}(t-\tau)) \frac{3}{2} B^{*} P_{1} \tilde{q}(t) \\
& +\left(\int_{-\infty}^{t} K(t-s) f(\tilde{q}(s)) d s\right)^{*} \frac{3}{2} C^{*} P_{1} \tilde{q}(t)-\tilde{q}^{*}(t) D^{T} P_{1} \tilde{q}(t) \\
& +\left(\int_{t-\delta}^{t} \tilde{q}(t) d s\right)^{*} D^{T} P_{1} D \tilde{q}(t)+\tilde{q}^{*}(t) D^{T} P_{1} D \int_{t-\delta}^{t} \tilde{q}(s) d s \\
& +\tilde{q}(t)^{*}(t) P_{2} \tilde{q}(t)-\tilde{q}^{*}(t-\delta) P_{2} \tilde{q}(t-\delta) \\
& +\delta^{2} \tilde{q}(t)^{*}(t) P_{3} \tilde{q}(t)-\left(\int_{t-\delta}^{t} \tilde{q}^{*}(s) d s\right)^{*} P_{3}\left(\int_{t-\delta}^{t} \tilde{q}^{*}(s) d s\right) \\
& +f^{*}(\tilde{q}(t)) P_{4} f(\tilde{q}(t))-f^{*}(\tilde{q}(t-\tau)) P_{4} f(\tilde{q}(t-\tau)) \\
& +\tilde{q}^{*}(t) L R L \tilde{q}(t)-\frac{1}{2} \tilde{q}^{*}(t-\delta) D^{T} P_{1} \tilde{q}(t) \\
& -\left(\int_{-\infty}^{t} K(s) f_{j}\left(\tilde{q}_{j}(t-s)\right) d s\right)^{*} R\left(\int_{-\infty}^{t} K(s) f_{j}\left(\tilde{q}_{j}(t-s)\right) d s\right) \\
& -\frac{1}{2} \tilde{q}^{*}(t) P_{1} D \tilde{q}(t-\delta)-\dot{\tilde{q}}^{*}(t) P_{1} \dot{\tilde{q}}(t)-\dot{\tilde{q}}^{*}(t) P_{1} D \tilde{q}(t-\delta) \\
& +\dot{\tilde{q}}^{*}(t) P_{1} A f(\check{q}(t))+\dot{\tilde{q}}^{*}(t) P_{1} B f(\tilde{q}(t-\tau)) \\
& +\dot{\tilde{q}}^{*}(t) P_{1} C \int_{-\infty}^{t} K(t-s) f(\tilde{q}(s)) d s \\
& -\left(\int_{t-\delta}^{t} \tilde{q}(s) d s\right)^{*} D^{T} P_{1} \dot{\tilde{q}}(t)-\left(\int_{t-\delta}^{t} \tilde{q}(s) d s\right)^{*} D^{T} P_{1} D \tilde{q}(t-\delta) \\
& \dot{\tilde{q}}^{*}(t) P_{1} \dot{\tilde{q}}(t)-\dot{\tilde{q}}(t-\delta) D^{T} P_{1} \dot{\tilde{q}}(t)-\dot{\tilde{q}}^{*}(t) P_{1} D \int_{t-\delta}^{t} \tilde{q}(s) d s \\
& \\
&
\end{aligned}
$$




$$
\begin{aligned}
& +f^{*}(\tilde{q}(t)) A^{*} P_{1} \int_{t-\delta}^{t} \tilde{q}(s) d s+f^{*}(\tilde{q}(t-\tau)) B^{*} P_{1} \int_{t-\delta}^{t} \tilde{q}(s) d s \\
& -\tilde{q}^{*}(t-\delta) D^{T} P_{1} D \int_{t-\delta}^{t} \tilde{q}(s) d s+\tilde{q}^{*}(t) L 2 P_{2} L \tilde{q}(t) \\
& -f^{*}(\tilde{q}(t)) 2 P_{2} f(\tilde{q}(t)) \\
= & \xi^{*}(\tilde{q}(t)) M \xi(\tilde{q}(t)),
\end{aligned}
$$

where $\xi^{*}(\tilde{q}(t))=\left[\tilde{q}^{*}(t), f^{*}(\tilde{q}(t)), f^{*}(\tilde{q}(t-\tau)),\left(\int_{-\infty}^{t} K(t-s) f(\tilde{q}(s)) d s\right)^{*},\left(\int_{t-\delta}^{t} \tilde{q}(s) d s\right)^{*},\left(\tilde{q}^{*}(t-\right.\right.$ $\left.\delta)), \dot{\tilde{q}}^{*}(t)\right]$.

Since $M<0$, we know

$$
\dot{V}(\tilde{q}(t)) \leq \xi^{*}(\tilde{q}(t)) M \xi(\tilde{q}(t)) \leq 0
$$

When $t=t_{k}, k=1,2, \ldots$, we define the impulsive function $M_{k}$ as follows:

$$
M_{k}\left(q\left(t_{k}^{-}\right), q_{t_{\bar{k}}^{-}}\right)=E_{k}\left[q\left(t_{k}^{-}\right)-\check{q}-D \int_{t_{k}-\delta}^{t_{k}}(q(s)-\check{q}) d s\right]
$$

where $k=1,2, \ldots, E_{k} \in \mathbb{H}^{n \times n}$. Meanwhile, we let

$$
\left(\begin{array}{cc}
P_{1} & \left(I+E_{k}\right)^{*} P_{1} \\
\star & P_{1}
\end{array}\right)>0,
$$

and then can compute

$$
\begin{aligned}
\left(\begin{array}{cc}
P_{1} & \left(I+E_{k}\right)^{*} P_{1} \\
\star & P_{1}
\end{array}\right)>0 & \Leftrightarrow\left(\begin{array}{cc}
I & 0_{1} \\
0 & P_{1}^{-1}
\end{array}\right)\left(\begin{array}{cc}
P_{1} & \left(I+E_{k}\right)^{*} P_{1} \\
\star & P_{1}
\end{array}\right)\left(\begin{array}{cc}
I & 0 \\
0 & P_{1}^{-1}
\end{array}\right)>0 \\
& \Leftrightarrow\left(\begin{array}{cc}
P_{1} & \left(I+E_{k}\right)^{*} \\
\star & P_{1}^{-1}
\end{array}\right)>0 \\
& \Leftrightarrow P_{1}-\left(I+E_{k}\right)^{*} P_{1}\left(I+E_{k}\right)>0,
\end{aligned}
$$

in which the last equivalence comes from Lemma 3. Thus, it yields

$$
\begin{aligned}
V_{1}\left(\tilde{q}\left(t_{k}\right)\right)= & \left(\tilde{q}\left(t_{k}\right)-D \int_{t_{k}-\delta}^{t_{k}} \tilde{q}(s) d s\right)^{*} P_{1}\left(\tilde{q}\left(t_{k}\right)-D \int_{t_{k}-\delta}^{t_{k}} \tilde{q}(s) d s\right) \\
= & \left(\tilde{q}\left(t_{k}^{-}\right)+E_{k}\left(\tilde{q}\left(t_{k}^{-}\right)-D \int_{t_{k}-\delta}^{t_{k}} \tilde{q}(s) d s\right)\right. \\
& \left.-D \int_{t_{k}-\delta}^{t_{k}} \tilde{q}(s) d s\right)^{*} \\
& +P_{1}\left(\tilde{q}\left(t_{k}^{-}\right)+E_{k}\left(\tilde{q}\left(t_{k}^{-}\right)-D \int_{t_{k}-\delta}^{t_{k}} \tilde{q}(s) d s\right)-D \int_{t_{k}-\delta}^{t_{k}} \tilde{q}(s) d s\right) \\
= & \left(\tilde{q}\left(t_{k}^{-}\right)-D \int_{t_{k}-\delta}^{t_{k}} \tilde{q}(s) d s\right)^{*}\left(I+E_{k}\right)^{*} P_{1}\left(I+E_{k}\right)\left(\tilde{q}\left(t_{k}^{-}\right)\right. \\
& \left.-D \int_{t_{k}-\delta}^{t_{k}} \tilde{q}(s) d s\right)
\end{aligned}
$$




$$
\begin{aligned}
& \leq\left(\tilde{q}\left(t_{k}^{-}\right)-D \int_{t_{k}-\delta}^{t_{k}} \tilde{q}(s) d s\right)^{*} P_{1}\left(\tilde{q}\left(t_{k}^{-}\right)-D \int_{t_{k}-\delta}^{t_{k}} \tilde{q}(s) d s\right) \\
& =V_{1}\left(\tilde{q}\left(t_{k}^{-}\right)\right) .
\end{aligned}
$$

Hence, we can infer that

$$
V\left(\tilde{q}\left(t_{k}\right)\right) \leq V\left(\tilde{q}\left(t_{k}^{-}\right)\right), \quad k=1,2, \ldots
$$

It follows from (41)-(44) that $V(\tilde{q}(t))$ is nonincreasing for $t \geq 0$. On the basis of assumption $\left(\mathbf{A}_{3}\right)$, letting $\int_{0}^{+\infty} s K(s) d s=\beta$, where $\beta$ is a nonnegative real constant, then, from the definition of $V(\tilde{q}(t))$, by assumption $\left(\mathbf{A}_{1}\right)$, together with Lemmas 4 and 5 , we can infer

$$
\begin{aligned}
& V(\tilde{q}(t)) \leq V(\tilde{q}(0))=\sum_{i=1}^{6} V_{i}(\tilde{q}(0)) \\
& =\left(\tilde{q}(0)-D \int_{-\delta}^{0} \tilde{q}(s) d s\right)^{*} P_{1}\left(\tilde{q}(0)-D \int_{-\delta}^{0} \tilde{q}(s) d s\right) \\
& +\int_{-\delta}^{0} \tilde{q}^{*}(s) P_{2} \tilde{q}(s) d s+\delta \int_{0}^{\delta} \int_{-u}^{0} \tilde{q}^{*}(s) P_{3} \tilde{q}(s) d s d u \\
& +\int_{-\tau}^{0} f^{*}(\tilde{q}(s)) P_{4} f(\tilde{q}(s)) d s \\
& +\int_{-\tau}^{0} \sum_{j=1}^{n} r_{j} \int_{0}^{\infty} K(s) \int_{-s}^{0} f_{j}^{*}\left(\tilde{q}_{j}(t)\right) f_{j}\left(\tilde{q}_{j}(t)\right) d t d s+\frac{1}{2} \tilde{q}^{*}(0) P_{1} \tilde{q}(0) \\
& =\tilde{q}^{*}(0) P_{1} \tilde{q}(0)-\tilde{q}^{*}(0) P_{1} D \int_{-\delta}^{0} \tilde{q}(s) d s-\left(\int_{-\delta}^{0} \tilde{q}(s) d s\right)^{*} D^{T} P_{1} \tilde{q}(0) \\
& +\left(\int_{-\delta}^{0} \tilde{q}(s) d s\right)^{*} D^{T} P_{1} D \int_{-\delta}^{0} \tilde{q}(s) d s+\int_{-\delta}^{0} \tilde{q}^{*}(s) P_{2} \tilde{q}(s) d s \\
& +\delta \int_{0}^{\delta} \int_{-u}^{0} \tilde{q}^{*}(s) P_{3} \tilde{q}(s) d s d u+\int_{-\tau}^{0} f^{*}(\tilde{q}(s)) P_{4} f(\tilde{q}(s)) d s \\
& +\sum_{j=1}^{n} r_{j} \int_{0}^{\infty} K(s) \int_{-s}^{0} f_{j}^{*}\left(\tilde{q}_{j}(t)\right) f_{j}\left(\tilde{q}_{j}(t)\right) d t d s+\frac{1}{2} \tilde{q}^{*}(0) P_{1} \tilde{q}(0) \\
& \leq\left(2 \lambda_{\max }\left(P_{1}\right)\left(1+\delta^{2}\right)+\delta \lambda_{\max }\left(P_{2}\right)+\frac{1}{2} \delta^{3} \lambda_{\max }\left(P_{3}\right)\right. \\
& +\tau \lambda_{\max }\left(L^{2}\right) \lambda_{\max }\left(P_{4}\right)+\beta \lambda_{\max }\left(L^{2}\right) \lambda_{\max }(R) \\
& \left.+\frac{1}{2} \lambda_{\max }\left(P_{1}\right)\right)\|\tilde{\vartheta}(t)\|^{2}
\end{aligned}
$$

Furthermore, by the definition of $V(\tilde{q}(t))$, we know

$$
V(\tilde{q}(t)) \geq V_{6}(\tilde{q}(t)) \geq \lambda_{\min }\left(P_{1}\right)\|\tilde{q}(t)\|^{2}
$$

According to (45) and (46), we obtain

$$
\|\tilde{q}(t)\| \leq \sqrt{\frac{U}{\lambda_{\min }\left(P_{1}\right)}}\|\tilde{\vartheta}(t)\|,
$$


where $U=2 \lambda_{\max }\left(P_{1}\right)\left(1+\delta^{2}\right)+\delta \lambda_{\max }\left(P_{2}\right)+\frac{1}{2} \delta^{3} \lambda_{\max }\left(P_{3}\right)+\tau \lambda_{\max }\left(L^{2}\right) \lambda_{\max }\left(P_{4}\right)+$ $\beta \lambda_{\max }\left(L^{2}\right) \lambda_{\max }(R)+\frac{1}{2} \lambda_{\max }\left(P_{1}\right)$.

Taking advantage of Lyapunov theory, we show that the equilibrium point $\check{q}$ of system (1) is globally robust stable.

The proof is completed.

Remark 1 Note that RVNNs and CVNNs are special cases of QVNNs. Therefore, RVNNs and CVNNs can also be considered in the results of this paper in the form of (1).

As LMI (3) is quaternion-valued, we should transform the quaternion LMI into complex one in order that it can be directly handled via the Matlab LMI toolbox. Hence, if we express the parameters as pairs of complex parts, i.e., $A_{0}^{R}+\imath A_{0}^{R}+\jmath A_{0}^{J}+\kappa A_{0}^{K}=A_{0}^{R}+\imath A_{0}^{R}+$ $\left(A_{0}^{J}+\imath A_{0}^{K}\right) \jmath=A_{1}+A_{2 \jmath}, B_{0}^{R}+\imath B_{0}^{R}+\jmath B_{0}^{J}+\kappa B_{0}^{K}=B_{0}^{R}+\imath B_{0}^{R}+\left(B_{0}^{J}+\imath B_{0}^{K}\right) \jmath=B_{1}+B_{2 \jmath}$, and $C_{0}^{R}+{ }_{\imath} C_{0}^{R}+{ }_{j} C_{0}^{J}+\kappa C_{0}^{K}=C_{0}^{R}+{ }_{\imath} C_{0}^{R}+\left(C_{0}^{J}+{ }_{\imath} C_{0}^{K}\right) J=C_{1}+C_{2 \jmath}$, where $A_{1}, A_{2}, B_{1}, B_{2}, C_{1}, C_{2} \in$ $\mathbb{C}^{n \times n}$, we can get the following result from Lemmas 7 and 8 .

Corollary 1 Suppose assumptions $\left(\mathbf{A}_{1}\right),\left(\mathbf{A}_{2}\right)$, and $\left(\mathbf{A}_{3}\right)$ are satisfied. If there exist a positive definite Hermitian matrix $P_{11} \in \mathbb{C}^{n \times n}$, skew-symmetric matrix $P_{12} \in \mathbb{C}^{n \times n}$, real positive diagonal matrices $P_{i} \in \mathbb{R}^{n \times n}(i=2,3,4)$ and $R$, positive constants $\lambda_{i}(i=1,2, \ldots, 27)$, such that following CVLMIs hold:

$$
\left(\begin{array}{cc}
P_{11} & -P_{12} \\
\bar{P}_{12} & \bar{P}_{1}
\end{array}\right)>0, \quad\left(\begin{array}{cc}
\Xi_{1} & -\Xi_{2} \\
\bar{\Xi}_{2} & \bar{\Xi}_{1}
\end{array}\right)<0,
$$

where $\Xi_{1}$ and $\Xi_{2}$ are defined in (48) and (49),

$$
\Xi_{1}=\left(\Xi_{i j}\right)_{41 \times 41}<0,
$$

where

$$
\begin{aligned}
& \Xi_{11}=-P_{11} D_{0}-D_{0}^{T} P_{11}+P_{2}+\delta^{2} P_{3}+L\left(2 P_{2}+R\right) L+\varepsilon_{1}\left(V_{D}\right)^{T} V_{D} \\
& +\varepsilon_{15}\left(V_{D}\right)^{T} V_{D}, \quad \Xi_{12}=\frac{3}{2}\left(P_{11} A_{1}-P_{12} \bar{A}_{2}\right), \\
& \Xi_{13}=\frac{3}{2}\left(P_{11} B_{1}-P_{12} \bar{B}_{2}\right), \quad \Xi_{14}=\frac{3}{2}\left(P_{11} C_{1}-P_{12} \bar{C}_{2}\right), \\
& \Xi_{15}=\left(D_{0}\right)^{T} P_{11} D_{0}, \quad \Xi_{16}=-\frac{1}{2} P_{11} D_{0}, \quad \Xi_{18}=P_{11} N_{D}, \quad \Xi_{19}=\frac{3}{2} P_{11} N_{A}^{R}, \\
& \Xi_{1.10}=\frac{3}{2} P_{11} N_{A}^{I}, \quad \Xi_{1.11}=\frac{3}{2} P_{11} N_{A}^{J}, \quad \Xi_{1.12}=\frac{3}{2} P_{11} N_{A}^{K}, \quad \Xi_{1.13}=\frac{3}{2} P_{11} N_{B}^{R}, \\
& \Xi_{1.14}=\frac{3}{2} P_{11} N_{B}^{I}, \quad \Xi_{1.15}=\frac{3}{2} P_{11} N_{B}^{J}, \quad \Xi_{1.16}=\frac{3}{2} P_{11} N_{B}^{K}, \quad \Xi_{1.17}=\frac{3}{2} P_{11} N_{C}^{R}, \\
& \Xi_{1.18}=\frac{3}{2} P_{11} N_{C}^{I}, \quad \Xi_{1.19}=\frac{3}{2} P_{11} N_{C}^{J}, \quad \Xi_{1.20}=\frac{3}{2} P_{11} N_{C}^{K}, \quad \Xi_{1.21}=D_{0}^{T} P_{11} N_{D}, \\
& \Xi_{1.23}=\left(U_{D} \Upsilon_{D} V_{D}\right)^{T} P_{11} N_{D}, \quad \Xi_{1.24}=\frac{1}{2} P_{11} N_{D}, \quad \Xi_{21}=\frac{3}{2} A_{1}^{*} P_{11}^{*}-A_{2}^{T} P_{12}^{*}, \\
& \Xi_{22}=P_{4}-2 P_{2}+\varepsilon_{2}\left(V_{A}^{R}\right)^{T} V_{A}^{R}+\varepsilon_{3}\left(V_{A}^{I}\right)^{T} V_{A}^{I}+\varepsilon_{4}\left(V_{A}^{J}\right)^{T} V_{A}^{J}+\varepsilon_{5}\left(V_{A}^{K}\right)^{T} V_{A}^{K}
\end{aligned}
$$




$$
\begin{aligned}
& +\varepsilon_{18}\left(V_{A}^{R}\right)^{T} V_{A}^{R}+\varepsilon_{19}\left(V_{A}^{I}\right)^{T} V_{A}^{I}+\varepsilon_{20}\left(V_{A}^{J}\right)^{T} V_{A}^{J}+\varepsilon_{21}\left(V_{A}^{K}\right)^{T} V_{A}^{K}, \\
& \Xi_{27}=A_{1}^{*} P_{11}+A_{2}^{T} \bar{P}_{12}, \quad \Xi_{31}=\frac{3}{2} B_{1}^{*} P_{11}^{*}-B_{2}^{T} P_{12}^{*}, \\
& \Xi_{33}=-P_{4}+\varepsilon_{6}\left(V_{B}^{R}\right)^{T} V_{B}^{R}+\varepsilon_{7}\left(V_{B}^{I}\right)^{T} V_{B}^{I}+\varepsilon_{8}\left(V_{B}^{J}\right)^{T} V_{B}^{J}+\varepsilon_{9}\left(V_{B}^{K}\right)^{T} V_{B}^{K} \\
& +\varepsilon_{22}\left(V_{B}^{R}\right)^{T} V_{B}^{R}+\varepsilon_{23}\left(V_{B}^{I}\right)^{T} V_{B}^{I}+\varepsilon_{24}\left(V_{B}^{J}\right)^{T} V_{B}^{J}+\varepsilon_{25}\left(V_{B}^{K}\right)^{T} V_{B}^{K}, \\
& \Xi_{37}=B_{1}^{*} P_{11}+B_{2}^{T} \bar{P}_{12}, \quad \Xi_{41}=\frac{3}{2} C_{1}^{*} P_{11}^{*}-C_{2}^{T} P_{12}^{*}, \\
& \Xi_{44}=-R+\varepsilon_{10}\left(V_{C}^{R}\right)^{T} V_{C}^{R}+\varepsilon_{11}\left(V_{C}^{I}\right)^{T} V_{C}^{I}+\varepsilon_{12}\left(V_{C}^{J}\right)^{T} V_{C}^{J}+\varepsilon_{13}\left(V_{C}^{K}\right)^{T} V_{C}^{K} \\
& +\varepsilon_{26}\left(V_{C}^{R}\right)^{T} V_{C}^{R}+\varepsilon_{27}\left(V_{C}^{I}\right)^{T} V_{C}^{I}+\varepsilon_{28}\left(V_{C}^{J}\right)^{T} V_{C}^{J}+\varepsilon_{29}\left(V_{C}^{K}\right)^{T} V_{C}^{K}, \\
& \Xi_{47}=C_{1}^{*} P_{11}+C_{2}^{T} \bar{P}_{12}, \quad \Xi_{51}=\left(D_{0}\right)^{T} P_{11} D_{0}, \\
& \Xi_{55}=-P_{3}+\varepsilon_{14}\left(V_{D}\right)^{T} V_{D}+\varepsilon_{16}\left(V_{D}\right)^{T} V_{D}+\varepsilon_{33}\left(V_{D}\right)^{T} V_{D}, \quad \Xi_{56}=-\left(D_{0}\right)^{T} P_{1} D_{0}, \\
& \Xi_{57}=-\left(D_{0}\right)^{T} P_{11}, \quad \Xi_{5.22}=D_{0}^{T} P_{11} N_{D}, \quad \Xi_{5.37}=P_{11} N_{D}, \quad \Xi_{5.39}=D_{0}^{T} P_{11} N_{D}, \\
& \Xi_{5.41}=\left(U_{D} \Upsilon_{D} V_{D}\right)^{T} P_{11} N_{D}, \quad \Xi_{61}=-\frac{1}{2} D_{0}^{T} P_{11}, \quad \Xi_{65}=-\left(D_{0}\right)^{T} P_{11} D_{0}, \\
& \Xi_{66}=-P_{2}+\varepsilon_{17}\left(V_{D}\right)^{T} V_{D}+\varepsilon_{32}\left(V_{D}\right)^{T} V_{D}+\varepsilon_{34}\left(V_{D}\right)^{T} V_{D}, \quad \Xi_{67}=-\left(D_{0}\right)^{T} P_{11}, \\
& \Xi_{6.38}=P_{11} N_{D}, \quad \Xi_{6.40}=D_{0}^{T} P_{11} N_{D}, \quad \Xi_{72}=P_{11}^{*} A_{1}+P_{12}^{T} \bar{A}_{2}, \\
& \Xi_{73}=P_{11}^{*} B_{1}+P_{12}^{T} \bar{B}_{2}, \quad \Xi_{74}=P_{11}^{*} C_{1}+P_{12}^{T} \bar{C}_{2}, \quad \Xi_{75}=-P_{11} D_{0}, \\
& \Xi_{76}=-P_{11} D_{0}, \quad \Xi_{77}=-P_{11}-P_{11}+\varepsilon_{30}\left(V_{D}\right)^{T} V_{D}+\varepsilon_{31}\left(V_{D}\right)^{T} V_{D}, \\
& \Xi_{7.25}=P_{11} N_{A}^{R}, \quad \Xi_{7.26}=P_{11} N_{A}^{I}, \quad \Xi_{7.27}=P_{11} N_{A}^{J}, \quad \Xi_{7.28}=P_{11} N_{A}^{K}, \\
& \Xi_{7.29}=P_{11} N_{B}^{R}, \quad \Xi_{7.30}=P_{11} N_{B}^{I}, \quad \Xi_{7.31}=P_{11} N_{B}^{J}, \quad \Xi_{7.32}=P_{11} N_{B}^{K}, \\
& \Xi_{7.33}=P_{11} N_{C}^{R}, \quad \Xi_{7.34}=P_{11} N_{C}^{I}, \quad \Xi_{7.35}=P_{11} N_{C}^{J}, \quad \Xi_{7.36}=P_{11} N_{C}^{K}, \\
& \Xi_{81}=\left(N_{D}\right)^{T} P_{11}, \quad \Xi_{88}=-\varepsilon_{1} I, \quad \Xi_{91}=\frac{3}{2}\left(N_{A}^{R}\right)^{T} P_{11}, \\
& \Xi_{99}=-\varepsilon_{2} I, \quad \Xi_{10.1}=\frac{3}{2}\left(N_{A}^{I}\right)^{T} P_{11}, \quad \Xi_{10.10}=-\varepsilon_{3} I, \\
& \Xi_{11.1}=\frac{3}{2}\left(N_{A}^{J}\right)^{T} P_{11}, \quad \Xi_{11.11}=-\varepsilon_{4} I, \quad \Xi_{12.1}=\frac{3}{2}\left(N_{A}^{K}\right)^{T} P_{11}, \quad \Xi_{12.12}=-\varepsilon_{5} I, \\
& \Xi_{13.1}=\frac{3}{2}\left(N_{B}^{R}\right)^{T} P_{11}, \quad \Xi_{13.13}=-\varepsilon_{6} I, \quad \Xi_{14.1}=\frac{3}{2}\left(N_{B}^{I}\right)^{T} P_{11}, \quad \Xi_{14.14}=-\varepsilon_{7} I, \\
& \Xi_{15.1}=\frac{3}{2}\left(N_{B}^{J}\right)^{T} P_{11}, \quad \Xi_{15.15}=-\varepsilon_{8} I, \quad \Xi_{16.1}=\frac{3}{2}\left(N_{B}^{K}\right)^{T} P_{11}, \quad \Xi_{16.16}=-\varepsilon_{9} I, \\
& \Xi_{17.1}=\frac{3}{2}\left(N_{C}^{R}\right)^{T} P_{11}, \quad \Xi_{17.17}=-\varepsilon_{10} I, \quad \Xi_{18.1}=\frac{3}{2}\left(N_{C}^{I}\right)^{T} P_{11}, \quad \Xi_{18.18}=-\varepsilon_{11} I, \\
& \Xi_{19.1}=\frac{3}{2}\left(N_{C}^{J}\right)^{T} P_{11}, \quad \Xi_{19.19}=-\varepsilon_{12} I, \quad \Xi_{20.1}=\frac{3}{2}\left(N_{C}^{K}\right)^{T} P_{11}, \quad \Xi_{20.20}=-\varepsilon_{13} I, \\
& \Xi_{21.1}=\left(N_{D}\right)^{T} P_{11} D_{0}, \quad \Xi_{21.21}=-\varepsilon_{14} I, \quad \Xi_{22.5}=\left(N_{D}\right)^{T} P_{11} D_{0}, \quad \Xi_{22.22}=-\varepsilon_{15} I, \\
& \Xi_{23.1}=\left(N_{D}\right)^{T} P_{11} U_{D} \Upsilon_{D} V_{D}, \quad \Xi_{23.23}=-\varepsilon_{16} I, \quad \Xi_{24.1}=\frac{1}{2}\left(N_{D}\right)^{T} P_{11}, \\
& \Xi_{24.24}=-\varepsilon_{17} I, \quad \Xi_{25.7}=\left(N_{A}^{R}\right)^{T} P_{11}, \quad \Xi_{25.25}=-\varepsilon_{18} I, \quad \Xi_{26.7}=\left(N_{A}^{I}\right)^{T} P_{11},
\end{aligned}
$$




$$
\begin{array}{llll}
\Xi_{26.26}=-\varepsilon_{19} I, & \Xi_{27.7}=\left(N_{A}^{J}\right)^{T} P_{11}, & \Xi_{27.27}=-\varepsilon_{20} I, & \Xi_{28.7}=\left(N_{A}^{K}\right)^{T} P_{11}, \\
\Xi_{28.28}=-\varepsilon_{21} I, & \Xi_{29.7}=\left(N_{B}^{R}\right)^{T} P_{11}, & \Xi_{29.29}=-\varepsilon_{22} I, & \Xi_{30.7}=\left(N_{B}^{I}\right)^{T} P_{11}, \\
\Xi_{30.30}=-\varepsilon_{23} I, & \Xi_{31.7}=\left(N_{B}^{J}\right)^{T} P_{11}, & \Xi_{31.31}=-\varepsilon_{24} I, & \Xi_{32.7}=\left(N_{B}^{K}\right)^{T} P_{11}, \\
\Xi_{32.32}=-\varepsilon_{25} I, & \Xi_{33.7}=\left(N_{C}^{R}\right)^{T} P_{11}, & \Xi_{33.33}=-\varepsilon_{26} I, & \Xi_{34.7}=\left(N_{C}^{I}\right)^{T} P_{11}, \\
\Xi_{34.34}=-\varepsilon_{27} I, & \Xi_{35.7}=\left(N_{C}^{J}\right)^{T} P_{11}, & \Xi_{35.35}=-\varepsilon_{28} I, & \Xi_{36.7}=\left(N_{C}^{K}\right)^{T} P_{11}, \\
\Xi_{36.36}=-\varepsilon_{29} I, & \Xi_{37.5}=\left(N_{D}\right)^{T} P_{11}, & \Xi_{37.37}=-\varepsilon_{30} I, & \Xi_{38.6}=\left(N_{D}\right)^{T} P_{11}, \\
\Xi_{38.38}=-\varepsilon_{31} I, & \Xi_{39.5}=\left(N_{D}\right)^{T} P_{11} D_{0}, \quad \Xi_{39.39}=-\varepsilon_{32} I, & \Xi_{40.6}=\left(N_{D}\right)^{T} P_{11} D_{0}, \\
\Xi_{40.40}=-\varepsilon_{33} I, & \Xi_{41.5}=\left(N_{D}\right)^{T} P_{11} U_{D} \Upsilon_{D} V_{D}, \quad \Xi_{41.41}=-\varepsilon_{34} I, \quad \text { and } \\
\Xi_{2}=\left(\Xi_{i j}\right)_{41 \times 41}<0, &
\end{array}
$$

where

$$
\begin{aligned}
& \Xi_{11}=-P_{12} D_{0}-D_{0}^{T} P_{12}, \quad \Xi_{12}=\frac{3}{2}\left(Q_{11} A_{2}+P_{12} \bar{A}_{1}\right), \quad \Xi_{13}=\frac{3}{2}\left(Q_{11} B_{2}+P_{12} \bar{B}_{1}\right), \\
& \Xi_{14}=\frac{3}{2}\left(Q_{11} C_{2}+P_{12} \bar{C}_{1}\right), \quad \Xi_{15}=\left(D_{0}\right)^{T} P_{12} D_{0}, \quad \Xi_{16}=-\frac{1}{2} P_{12} D_{0}, \\
& \Xi_{18}=P_{12} N_{D}, \quad \Xi_{19}=\frac{3}{2} P_{12} N_{A}^{R}, \quad \Xi_{1.10}=\frac{3}{2} P_{12} N_{A}^{I}, \quad \Xi_{1,11}=\frac{3}{2} P_{12} N_{A}^{J}, \\
& \Xi_{1.12}=\frac{3}{2} P_{12} N_{A}^{K}, \quad \Xi_{1.13}=\frac{3}{2} P_{12} N_{B}^{R}, \quad \Xi_{1.14}=\frac{3}{2} P_{12} N_{B}^{I}, \quad \Xi_{1.15}=\frac{3}{2} P_{12} N_{B}^{J}, \\
& \Xi_{1.16}=\frac{3}{2} P_{12} N_{B}^{K}, \quad \Xi_{1.17}=\frac{3}{2} P_{12} N_{C}^{R}, \quad \Xi_{1.18}=\frac{3}{2} P_{12} N_{C}^{I}, \quad \Xi_{1.19}=\frac{3}{2} P_{12} N_{C}^{J}, \\
& \Xi_{1.20}=\frac{3}{2} P_{12} N_{C}^{K}, \quad \Xi_{1.21}=D_{0}^{T} P_{12} N_{D}, \quad \Xi_{1.23}=\left(U_{D} \Upsilon_{D} V_{D}\right)^{T} P_{12} N_{D}, \\
& \Xi_{1.24}=\frac{1}{2} P_{12} N_{D}, \quad \Xi_{21}=-\frac{3}{2} A_{2}^{T} P_{11}^{T}-A_{1}^{*} P_{12}^{T}, \quad \Xi_{27}=A_{1}^{*} P_{12}-A_{2}^{T} \bar{P}_{11}, \\
& \Xi_{31}=-\frac{3}{2} B_{2}^{T} P_{11}^{T}-B_{1}^{T} P_{12}^{T}, \quad \Xi_{37}=B_{1}^{*} P_{11}+B_{2}^{T} \bar{P}_{12}, \quad \Xi_{41}=-\frac{3}{2} C_{2}^{T} P_{11}^{T}-C_{1}^{T} P_{12}^{T}, \\
& \Xi_{47}=C_{1}^{*} P_{11}+C_{2}^{T} \bar{P}_{12}, \quad \Xi_{51}=\left(D_{0}\right)^{T} P_{12} D_{0}, \quad \Xi_{56}=-\left(D_{0}\right)^{T} P_{12} D_{0}, \\
& \Xi_{57}=-\left(D_{0}\right)^{T} P_{12}, \quad \Xi_{5.22}=D_{0}^{T} P_{12} N_{D}, \quad \Xi_{5.37}=P_{12} N_{D}, \\
& \Xi_{5.39}=D_{0}^{T} P_{12} N_{D}, \quad \Xi_{5.41}=\left(U_{D} \Upsilon_{D} V_{D}\right)^{T} P_{12} N_{D}, \quad \Xi_{61}=-\frac{1}{2} D_{0}^{T} P_{12}, \\
& \Xi_{65}=-\left(D_{0}\right)^{T} P_{12} D_{0}, \quad \Xi_{67}=-\left(D_{0}\right)^{T} P_{12}, \quad \Xi_{6.38}=P_{12} N_{D}, \quad \Xi_{6.40}=D_{0}^{T} P_{12} N_{D}, \\
& \Xi_{72}=P_{11}^{*} A_{1}+P_{12}^{T} \bar{A}_{2}, \quad \Xi_{73}=P_{11}^{*} B_{1}+P_{12}^{T} \bar{B}_{2}, \quad \Xi_{74}=P_{11}^{*} C_{1}+P_{12}^{T} \bar{C}_{2}, \\
& \Xi_{75}=-P_{11} D_{0}, \quad \Xi_{76}=-P_{11} D_{0}, \quad \Xi_{77}=-P_{12}-P_{12}, \quad \Xi_{7.25}=P_{12} N_{A}^{R}, \\
& \Xi_{7.26}=P_{12} N_{A}^{I}, \quad \Xi_{7.27}=P_{12} N_{A}^{J}, \quad \Xi_{7.28}=P_{12} N_{A}^{K}, \quad \Xi_{7.29}=P_{12} N_{B}^{R} \text {, } \\
& \Xi_{7.30}=P_{12} N_{B}^{I}, \quad \Xi_{7.31}=P_{12} N_{B}^{J}, \quad \Xi_{7.32}=P_{12} N_{B}^{K}, \quad \Xi_{7.33}=P_{12} N_{C}^{R}, \\
& \Xi_{7.34}=P_{12} N_{C}^{I}, \quad \Xi_{7.35}=P_{12} N_{C}^{J}, \quad \Xi_{7.36}=P_{12} N_{C}^{K}, \quad \Xi_{81}=-\left(N_{D}\right)^{T} P_{12}, \\
& \Xi_{91}=-\frac{3}{2}\left(N_{A}^{R}\right)^{T} P_{12}, \quad \Xi_{10.1}=-\frac{3}{2}\left(N_{A}^{I}\right)^{T} P_{12}, \quad \Xi_{11.1}=-\frac{3}{2}\left(N_{A}^{J}\right)^{T} P_{12},
\end{aligned}
$$




$$
\begin{array}{lll}
\Xi_{12.1}=-\frac{3}{2}\left(N_{A}^{K}\right)^{T} P_{12}, & \Xi_{13.1}=-\frac{3}{2}\left(N_{B}^{R}\right)^{T} P_{12}, & \Xi_{14.1}=-\frac{3}{2}\left(N_{B}^{I}\right)^{T} P_{12}, \\
\Xi_{15.1}=-\frac{3}{2}\left(N_{B}^{J}\right)^{T} P_{12}, & \Xi_{16.1}=-\frac{3}{2}\left(N_{B}^{K}\right)^{T} P_{12}, & \Xi_{17.1}=-\frac{3}{2}\left(N_{C}^{R}\right)^{T} P_{12}, \\
\Xi_{18.1}=-\frac{3}{2}\left(N_{C}^{I}\right)^{T} P_{12}, & \Xi_{19.1}=-\frac{3}{2}\left(N_{C}^{J}\right)^{T} P_{12}, & \Xi_{20.1}=-\frac{3}{2}\left(N_{C}^{K}\right)^{T} P_{12}, \\
\Xi_{21.1}=-\left(N_{D}\right)^{T} P_{12} D_{0}, & \Xi_{22.5}=-\left(N_{D}\right)^{T} P_{12} D_{0}, & \Xi_{23.1}=-\left(N_{D}\right)^{T} P_{12} U_{D} \Upsilon_{D} V_{D}, \\
\Xi_{24.1}=-\frac{1}{2}\left(N_{D}\right)^{T} P_{12}, & \Xi_{25.7}=-\left(N_{A}^{R}\right)^{T} P_{12}, & \Xi_{26.7}=-\left(N_{A}^{I}\right)^{T} P_{12}, \\
\Xi_{27.7}=-\left(N_{A}^{J}\right)^{T} P_{12}, & \Xi_{28.7}=-\left(N_{A}^{K}\right)^{T} P_{12}, & \Xi_{29.7}=-\left(N_{B}^{R}\right)^{T} P_{12}, \\
\Xi_{30.7}=-\left(N_{B}^{I}\right)^{T} P_{12}, & \Xi_{31.7}=-\left(N_{B}^{J}\right)^{T} P_{12}, & \Xi_{32.7}=-\left(N_{B}^{K}\right)^{T} P_{12}, \\
\Xi_{33.7}=-\left(N_{C}^{R}\right)^{T} P_{12}, & \Xi_{34.7}=-\left(N_{C}^{I}\right)^{T} P_{12}, & \Xi_{35.7}=-\left(N_{C}^{J}\right)^{T} P_{12}, \\
\Xi_{36.7}=-\left(N_{C}^{K}\right)^{T} P_{12}, & \Xi_{37.5}=-\left(N_{D}\right)^{T} P_{12}, & \Xi_{38.6}=-\left(N_{D}\right)^{T} P_{12}, \\
\Xi_{39.5}=-\left(N_{D}\right)^{T} P_{12} D_{0}, & \Xi_{40.6}=-\left(N_{D}\right)^{T} P_{12} D_{0}, & \Xi_{41.5}=-\left(N_{D}\right)^{T} P_{12} U_{D} \Upsilon_{D} V_{D},
\end{array}
$$

and the other entries in $\Xi_{1}$ and $\Xi_{2}$ are zeros.

Proof According to Lemmas 7 and 8, as well as Theorem 1, we can easily have the result.

\section{Numerical example}

In this section, we illustrate the validity of our results with the following example.

Example 1 Take the parameters of QVNN as follows:

$$
\begin{aligned}
& \check{D}=\left(\begin{array}{cc}
0.029 & 0 \\
0 & 0.0305
\end{array}\right), \quad \hat{D}=\left(\begin{array}{cc}
0.032 & 0 \\
0 & 0.0307
\end{array}\right), \\
& L=\left(\begin{array}{cc}
0.005 & 0 \\
0 & 0.005
\end{array}\right), \quad \delta=0.05, \quad \tau=0.1, \\
& E_{k}=\left(\begin{array}{cc}
-0.3+0.2 i & 0 \\
0.1-0.6 i & -0.2+0.2 i
\end{array}\right), \quad k \in 1,2, \ldots, \\
& \check{A}=\left(\check{a}_{i j}\right)_{2 \times 2}, \quad \check{A}=\left(\check{a}_{i j}\right)_{2 \times 2}, \quad \check{B}=\left(\check{b}_{i j}\right)_{2 \times 2}, \\
& \check{B}=\left(\check{b}_{i j}\right)_{2 \times 2}, \quad \check{C}=\left(\check{c}_{i j}\right)_{2 \times 2}, \quad \check{C}=\left(\check{c}_{i j}\right)_{2 \times 2},
\end{aligned}
$$

where

$$
\begin{aligned}
& \check{a}_{11}=-0.002-0.002 \imath+0.002 \jmath+0 \kappa, \\
& \check{a}_{12}=-0.001-0.001 \imath-0.001 J-0.001 \kappa, \\
& \check{a}_{21}=0.001-0.001 \imath-0.001 \jmath-0.001 \kappa, \\
& \check{a}_{22}=-0.0015-0.002 \imath-0.002 \jmath-0.002 \kappa, \\
& \hat{a}_{11}=0.001+0.001 \imath+0.001 \jmath+0.001 \kappa, \quad \hat{a}_{12}=0+0 \imath+0 J+0 \kappa,
\end{aligned}
$$




$$
\begin{aligned}
& \hat{a}_{21}=0.0015+0 \imath+0 J+0 \kappa, \\
& \hat{a}_{22}=0.001+0.001 \imath+0.001 \jmath+0.001 \kappa, \\
& \check{b}_{11}=-0.002-0.001 l-0.001 J-0.001 \kappa \text {, } \\
& \check{b}_{12}=-0.001-0.001 l-0.001 J-0.001 \kappa \text {, } \\
& \check{b}_{21}=-0.001-0.001 l-0.001 J-0.001 \kappa, \\
& \check{b}_{22}=-0.001-0.001 l-0.001 J-0.001 \kappa \text {, } \\
& \hat{b}_{11}=0.001+0.001 \imath+0.001 J+0.001 \kappa, \quad \hat{b}_{12}=0+0 \imath+0 \jmath+0 \kappa, \\
& \hat{b}_{21}=0+0 \imath+0 \jmath+0 \kappa, \quad \hat{b}_{22}=0.001+0.001 \imath+0.001 \jmath+0.001 \kappa, \\
& \check{c}_{11}=0.001-0.001 \imath-0.0015 J-0.001 \kappa \text {, } \\
& \check{c}_{12}=-0.001-0.001 l-0.0014 J-0.001 \kappa \text {, } \\
& \check{c}_{21}=-0.001-0.001 \imath-0.001 \jmath-0.001 \kappa, \\
& \check{c}_{22}=-0.001-0.001 l-0.001 J-0.001 \kappa \text {, } \\
& \hat{c}_{11}=0.0015+0.0034 l+0.001 J+0.0015 \kappa, \quad \hat{c}_{12}=0+0.0012 \imath+0 J+0 \kappa, \\
& \hat{c}_{21}=0+0.0041 \imath+0.001 \jmath+0.0015 \kappa, \quad \hat{c}_{22}=0.0012+0.001 \imath+0 J+0.002 \kappa .
\end{aligned}
$$

In addition, we take activation and delay kernel functions as follows:

$$
\begin{aligned}
& f_{1}(q)=f_{2}(q)=(|q+1|-|q-1|) \times 0.1, \quad \forall q=q_{0}+i q_{1}+j q_{2}+k q_{3} \in \mathbb{H} . \\
& K_{1}(s)=K_{2}(s)=e^{-s}, \quad s \in[0,+\infty) .
\end{aligned}
$$

Using MATLAB tools, we can get the results for the LMI (3) in Theorem 1:

$$
\begin{aligned}
& P_{1}=\left(\begin{array}{cc}
44.3253+0.0000 \imath & -1.0086+1.1303 \imath \\
-1.0086-1.1303 \imath & 46.9798+0.0000 \imath
\end{array}\right), \\
& P_{2}=\left(\begin{array}{cc}
1.2253 & 0 \\
0 & 1.3223
\end{array}\right), \quad P_{3}=\left(\begin{array}{cc}
7.7262 & 0 \\
0 & 12.9308
\end{array}\right), \\
& P_{4}=\left(\begin{array}{cc}
1.1407 & 0 \\
0 & 1.1596
\end{array}\right), \quad R=\left(\begin{array}{cc}
79.5482 & 0 \\
0 & 80.5706
\end{array}\right), \\
& \varepsilon_{1}=49.8632, \quad \varepsilon_{2}=112.5925, \quad \varepsilon_{3}=112.5168, \quad \varepsilon_{4}=112.5168, \\
& \varepsilon_{5}=108.2435, \quad \varepsilon_{6}=112.5942, \quad \varepsilon_{7}=110.3803, \quad \varepsilon_{8}=110.3803, \\
& \varepsilon_{9}=110.3803, \quad \varepsilon_{10}=144.5979, \quad \varepsilon_{11}=218.7847, \quad \varepsilon_{12}=170.7464, \\
& \varepsilon_{13}=179.4117, \quad \varepsilon_{14}=156.2501, \quad \varepsilon_{15}=18.9679, \quad \varepsilon_{16}=75.6929, \\
& \varepsilon_{17}=46.0796, \quad \varepsilon_{18}=33.2054, \quad \varepsilon_{19}=30.5856, \quad \varepsilon_{20}=30.5856, \\
& \varepsilon_{21}=37.6041, \quad \varepsilon_{22}=32.8740, \quad \varepsilon_{23}=36.5195, \quad \varepsilon_{24}=36.5195, \\
& \varepsilon_{25}=36.5195, \quad \varepsilon_{26}=76.0829, \quad \varepsilon_{27}=76.1625, \quad \varepsilon_{28}=76.1027,
\end{aligned}
$$




$$
\begin{aligned}
& \varepsilon_{29}=76.1166, \quad \varepsilon_{30}=76.1752, \quad \varepsilon_{31}=98.0675, \\
& \varepsilon_{32}=31.7173, \quad \varepsilon_{33}=75.7220, \quad \varepsilon_{34}=31.7172 .
\end{aligned}
$$

Obviously, it satisfies the conditions of Theorem 1, and there exists a unique equilibrium point which is globally robust and stable.

Next, we select the fixed neural network parameters:

$$
\begin{aligned}
& D=\left(\begin{array}{cc}
0.03 & 0 \\
0 & 0.0306
\end{array}\right), \quad A=\left(a_{i j}\right)_{2 \times 2}, \quad B=\left(b_{i j}\right)_{2 \times 2}, \quad C=\left(c_{i j}\right)_{2 \times 2}, \\
& J=\left(\begin{array}{cc}
0.001-0.001 \imath-0.002 \jmath+0.005 \kappa & 0 \\
0 & -0.002+0.001 \imath+0 \jmath-0.001 \kappa
\end{array}\right),
\end{aligned}
$$

where

$$
\begin{aligned}
& a_{11}=-0.001-0.001 \imath+0 \jmath+0.0005 \kappa, \\
& a_{12}=-0.001-0.0005 \imath-0.0007 \jmath-0.0005 \kappa, \\
& a_{21}=0.01-0.0005 \imath-0.0005 \jmath+0 \kappa, \quad a_{22}=-0.001+0 \imath+0 \jmath+0 \kappa, \\
& b_{11}=-0.001+0.001 \imath+0 \jmath+0 \kappa, \quad b_{12}=0+0 \imath-0.001 \jmath-0.001 \kappa, \\
& b_{21}=0-0.0005 \imath-0.0005 \jmath-0.0005 \kappa, \quad b_{22}=0.0005+0 \imath+0 \jmath+0 \kappa, \\
& c_{11}=0.0012+0.002 \imath+0 \jmath+0.001 \kappa, \\
& c_{12}=-0.0005-0.0006 \imath-0.001 \jmath-0.001 \kappa, \\
& c_{21}=0.0005+0.002 \imath-0 \jmath-0 \kappa, \quad c_{22}=0.001+0 \imath-0.0005 \jmath+0.001 \kappa .
\end{aligned}
$$

By employing Quaternion Toolbox for Matlab and the fourth-order Runge-Kutta method, we perform numerical simulation of the network. Figures 1, 2, 3, and 4 depict the four parts of the states of the considered system, where the initial conditions are chosen by 10 random constant quaternion-valued vectors. It can be seen from these figures that each neuron state converges to the stable equilibrium point, which is $(0.0338-0.0322 \imath-0.0657 \jmath+$ $0.1680 \kappa,-0.0634-0.0323 \imath-0.0004 \jmath-0.0331 \kappa)^{T}$.

\section{Conclusions}

In this paper, the issue of robust stability of pulse and delay QVNNs with interval parameter uncertainties is considered. A sufficient condition to guarantee the existence, uniqueness, and global robust stability of an equilibrium point has been deduced by using homomorphic mapping theorem, Lyapunov method, and inequality techniques. Finally, the effectiveness of the proposed theoretical condition is verified via a numerical example. It should be noted that the activation function is continuous in this article, however, the discontinuous case in QVNNs is our coming consideration, which is of great importance in real applications of everyday life.

Recently, some researchers investigated the Mittag-Leffler stability of multiple equilibrium points for fractional-order QVNNs with an impulsive term [33]. Other interesting 


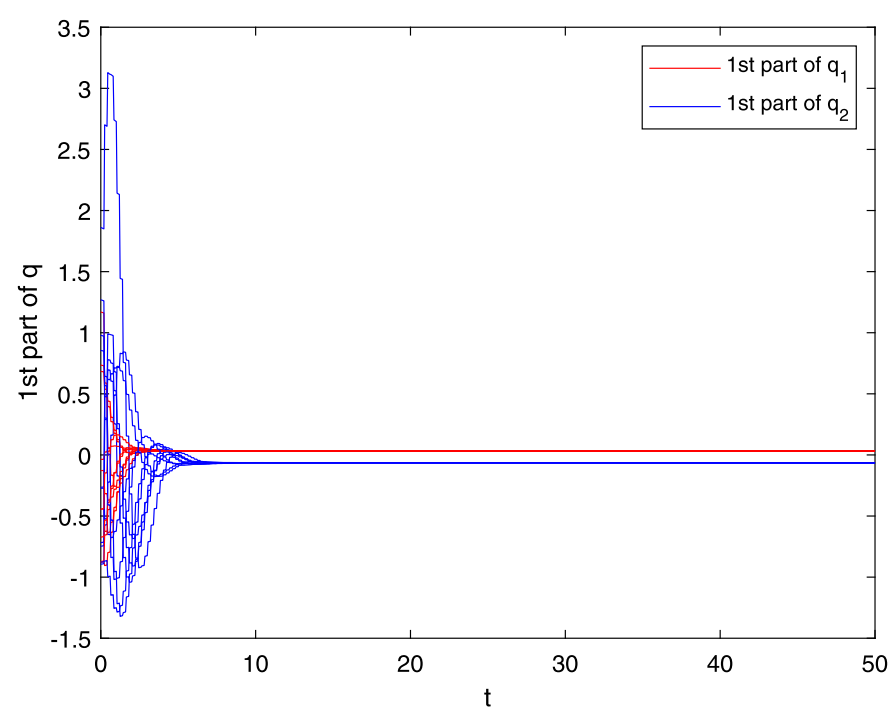

Figure 1 The first part of the state trajectories for system (1) with parameters

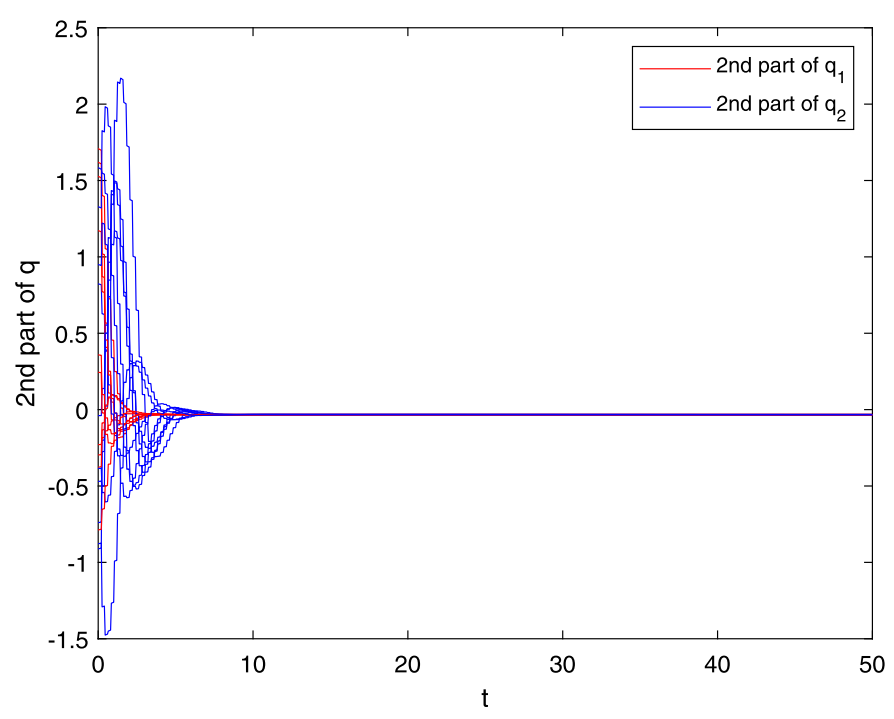

Figure 2 The second part of the state trajectories for system (1) with parameters

work involves the Hopf bifurcation of a fractional-order octonion-valued neural networks with time delay [34]. This important work is a substantial extension of traditional integerorder neural networks, which provide us a new way to extend our work. Note that, in references [33] and [34], to obtain the corresponding results, the authors transform their QVNNs into several RVNNs or CVNNs from research methods. In this article, we obtain the dynamical behaviors of QVNNs directly using the basic properties of QVNNs, instead of converting them into complex- or real-valued system, which avoids the increase of system dimension. 


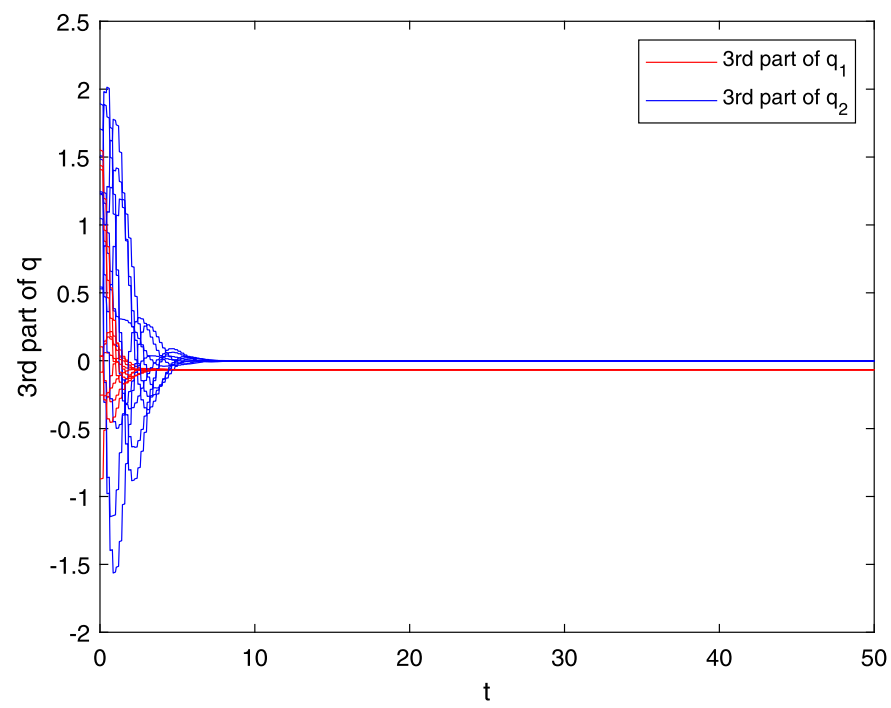

Figure 3 The third part of the state trajectories for system (1) with parameters

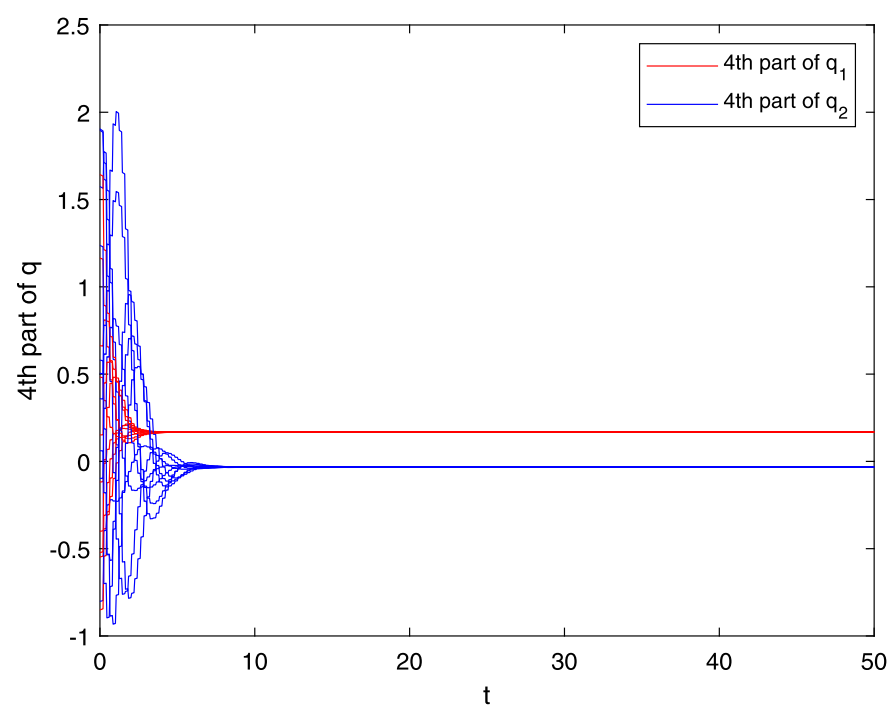

Figure 4 The fourth part of the state trajectories for system (1) with parameters

Acknowledgements

We are thankful to the editor and the anonymous reviewers for many valuable suggestions to improve this paper.

Funding

This work was supported by the National Natural Science Foundation of China under Grants $(11961024,11801047)$, the Natural Science Foundation of Chongqing under Grant (cstc2019jcyj-msxmX0755, cstc2017jcyjAX0131,

cstc2018jcyjAX0606), the Science and Technology Research Program of the Chongqing Municipal Education Commission under Grant KJQN201900701.

Availability of data and materials

Data sharing not applicable to this article as no data sets were generated or analyzed during the current study.

Competing interests

The authors declare that they have no conflict of interest. 
Authors' contributions

All authors conceived of the study, participated in its design and coordination, read and approved the final manuscript.

\section{Publisher's Note}

Springer Nature remains neutral with regard to jurisdictional claims in published maps and institutional affiliations.

\section{Received: 9 June 2020 Accepted: 21 October 2020 Published online: 07 January 2021}

\section{References}

1. Ding, D., Wang, Z., Shen, B., Dong, H.: Envelope-constrained $H_{\infty}$ filtering with fading measurements and randomly occurring nonlinearities: the finite horizon case. Automatica 55, 37-45 (2015)

2. Dong, H., Wang, Z., Ding, S.X., Gao, H.: Finite-horizon estimation of randomly occurring faults for a class of nonlinear time-varying systems. Automatica 50, 3182-3189 (2014)

3. Chen, T., Wang, L.: Global $\mu$-stability of delayed neural networks with unbounded time-varying delays. IEEE Trans. Neural Netw. 18(6), 1836-1840 (2007)

4. Chen, X., Song, Q., Li, Z:: Design and analysis of quaternion-valued neural networks for associative memories. IEEE Trans. Syst. Man Cybern. Syst. 99, 1-10 (2017)

5. Chen, T., Wang, L.: Power-rate global stability of dynamical systems with unbounded time-varying delays. IEEE Trans. Circuits Syst. II, Express Briefs 54(8), 705-709 (2007)

6. Hirose, A.: Proposal of fully complex-valued neural networks. Int. Jt. Conf. Neural Netw. 4, 152-157 (1992)

7. Jian, J., Zhao, Z:: Global stability in Lagrange sense for BAM-type Cohen-Grossberg neural networks with time-varying delays. Syst. Sci. Control Eng. 3, 1-7 (2015)

8. Song, Q., Yu, Q., Zhao, Z., Liu, Y., Alsaadi, F.E.: Boundedness and global robust stability analysis of delayed complex-valued neural networks with interval parameter uncertainties. Neural Netw. 103, 55-62 (2018)

9. Duan, C., Song, Q.: Boundedness and stability for discrete-time delayed neural network with complex-valued linear threshold neurons. Discrete Dyn. Nat. Soc. 2010, Article ID 368379 (2010)

10. Yang, X., Li, C., Huang, T., Song, Q., Huang, J.: Global Mittag-Leffler synchronization of fractional-order neural networks via impulsive control. Neural Process. Lett. 48(1), 459-479 (2017)

11. Li, Q., Liang, J.: Dissipativity of the stochastic Markovian switching CVNNs with randomly occurring uncertainties and general uncertain transition rates. Int. J. Syst. Sci. 51, 1102-1118 (2020)

12. Li, Q., Liang, J., Gong, W.: Stabilization of piecewise-homogeneous Markovian switching CVNNs with mode-dependent delays and incomplete transition rates. Syst. Sci. Control Eng. 8(1), 206-221 (2020)

13. Tan, Y., Tang, S., Chen, X.: Robust stability analysis of impulsive complex-valued neural networks with time delays and parameter uncertainties. J. Inequal. Appl. 2017, 215 (2017)

14. Tan, Y., Tang, S., Chen, X.: Robust stability analysis of impulsive complex-valued neural networks with mixed time delays and parameter uncertainties. Adv. Differ. Equ. 2018(1), 62 (2018)

15. Minemoto, T., Isokawa, T., Nishimura, H.: Quaternionic multistate Hopfield neural network with extended projection rule. Artif. Life Robot. 21(1), 106-111 (2016)

16. Li, Y., Qin, J.: Existence and global exponential stability of periodic solutions for quaternion-valued cellular neural networks with time-varying delays. Neurocomputing 292, 91-103 (2018)

17. Liu, Y., Zhang, D., Lu, J., Cao, J.: Global $\mu$-stability criteria for quaternion-valued neural networks with unbounded time-varying delays. Inf. Sci. 360, 273-288 (2016)

18. Zhu, J., Sun, J.: Stability of quaternion-valued impulsive delay difference systems and its application to neural networks. Neurocomputing 284(5), 63-69 (2018)

19. Liu, Y., Zhang, D., Lu, J.: Global exponential stability for quaternion-valued recurrent neural networks with time-varying delays. Nonlinear Dyn. 87(1), 553-565 (2016)

20. Kusamichi, H., Isokawa, T., Matsui, N.: A new scheme for color night vision by quaternion neural network. In: Proc. 2nd Int. Conf. Auton. Robots Agents, pp. 101-106 (2004)

21. Isokawa, T., Nishimura, H., Kamiura, N.: Associative memory in quaternionic Hopfield neural network. Int. J. Neural Syst. 18(2), 135-145 (2008)

22. Tu, Z., Zhao, Y., Ding, N., Feng, Y., Zhang, W.: Stability analysis of quaternion-valued neural networks with both discrete and distributed delays. Appl. Math. Comput. 343, 342-353 (2019)

23. Li, X., Fu, X., Balasubramaniam, P., Rakkiyappan, R.: Existence, uniqueness and stability analysis of recurrent neural networks with time delay in the leakage term under impulsive perturbations. Nonlinear Anal. 11(5), 4092-4108 (2010)

24. Gopalsamy, K.: Leakage delays in BAM. J. Math. Anal. Appl. 325(2), 1117-1132 (2007)

25. Chen, X., Song, Q.: Global stability of complex-valued neural networks with both leakage time delay and discrete time delay on time scales. Neurocomputing 121, 254-264 (2013)

26. Yu, W.W., Cao, J.D.: Synchronization control of stochastic delayed neural networks. Physica A 373, $252-260$ (2007)

27. Song, Q., Zhao, Z.: Stability criterion of complex-valued neural networks with both leakage delay and time-varying delays on time scales. Neurocomputing 171, 179-184 (2016)

28. Wang, X., Zhou, J., Chen, X., Tan, Y.: Parameter-range-dependent robust stability conditions for quaternion-valued neural networks with time delays. Adv. Differ. Equ. 2019(1), 181 (2019)

29. Zhang, W., Tang, Y., Miao, Q., Du, W.: Exponential synchronization of coupled switched neural networks with mode-dependent impulsive effects. IEEE Trans. Neural Netw. Learn. Syst. 24(8), 1316-1326 (2013)

30. Zhang, W., Tang, Y., Wu, X., Fang, J.A.: Synchronization of nonlinear dynamical networks with heterogeneous impulses. IEEE Trans. Circuits Syst. I, Regul. Pap. 61(4), 1220-1228 (2014)

31. Gopalsamy, K.: Stability of artificial neural networks with impulses. Appl. Math. Comput. 154(3), 783-813 (2004)

32. Rakkiyappan, R., Balasubramaniam, P., Cao, J.: Global exponential stability results for neutral type impulsive neural networks. Nonlinear Anal. 11(1), 122-130 (2010) 
33. Udhayakumar, K.: Mittag-Leffler stability analysis of multiple equilibrium points in impulsive fractional-order quaternion-valued neural networks. Front. Inf. Technol. Electron. Eng. 21(2), 234-246 (2020)

34. Kandasamy, U., Rajan, R.: Hopf bifurcation of a fractional-order octonion-valued neural networks with time delays. Discrete Contin. Dyn. Syst., Ser. S 13(9), 2537-2559 (2020)

35. Chen, X., Li, Z., Song, Q., Hu, J., Tan, Y.: Robust stability analysis of quaternion-valued neural networks with time delays and parameter uncertainties. Neural Netw. 91, 55-65 (2017)

36. Chen, X., Li, L., Li, Z.: Robust stability analysis of quaternion-valued neural networks via LMI approach. Adv. Differ. Equ. 2018(1), $131(2018)$

37. Chen, X., Song, Q.: State estimation for quaternion-valued neural networks with multiple time delays. IEEE Trans. Syst. Man Cybern. Syst. 49(11), 2278-2287 (2019)

Submit your manuscript to a SpringerOpen ${ }^{0}$ journal and benefit from:

- Convenient online submission

- Rigorous peer review

- Open access: articles freely available online

- High visibility within the field

- Retaining the copyright to your article

Submit your next manuscript at $\gg$ springeropen.com 